\title{
Faint objects in motion: the new frontier of high precision astrometry
}

\section{Fabien Malbet, et al. [full author details at the end of the article]}

Received: 1 August 2020 / Accepted: 15 March 2021 / Published online: 4 September 2021

(C) The Author(s) 2021

\begin{abstract}
Sky survey telescopes and powerful targeted telescopes play complementary roles in astronomy. In order to investigate the nature and characteristics of the motions of very faint objects, a flexibly-pointed instrument capable of high astrometric accuracy is an ideal complement to current astrometric surveys and a unique tool for precision astrophysics. Such a space-based mission will push the frontier of precision astrometry from evidence of Earth-mass habitable worlds around the nearest stars, to distant Milky Way objects, and out to the Local Group of galaxies. As we enter the era of the James Webb Space Telescope and the new ground-based, adaptive-optics-enabled giant telescopes, by obtaining these high precision measurements on key objects that Gaia could not reach, a mission that focuses on high precision astrometry science can consolidate our theoretical understanding of the local Universe, enable extrapolation of physical processes to remote redshifts, and derive a much more consistent picture of cosmological evolution and the likely fate of our cosmos. Already several missions have been proposed to address the science case of faint objects in motion using high precision astrometry missions: NEAT proposed for the ESA M3 opportunity, micro-NEAT for the S1 opportunity, and Theia for the M4 and M5 opportunities. Additional new mission configurations adapted with technological innovations could be envisioned to pursue accurate measurements of these extremely small motions. The goal of this White Paper is to address the fundamental science questions that are at stake when we focus on the motions of faint sky objects and to briefly review instrumentation and mission profiles.
\end{abstract}

Keywords Astrometry $\cdot$ Cosmology $\cdot$ Local universe $\cdot$ Exoplanets $\cdot$ Space mission

This paper is the accurate transcription with a very few updates of the White Paper called Faint objects in motion: the new frontier of high precision astrometry

Fabien Malbet

fabien.malbet@univ-grenoble-alpes.fr

Alessandro Sozzetti

alessandro.sozzetti@inaf.it 
submitted in 2019 to the call for the next planning cycle of the ESA Science Programme, called "Voyage 2050". Following ESA instructions, the main aim of this paper is to argue why high precision astrometry should have priority in the Voyage 2050 planning cycle. In order to ensure realism in the resulting Programme, we were asked to illustrate possible mission profiles which is possible thanks to the previous work on the proposed space missions. Therefore, this paper focusses on the scientific issues where most figures refer to the Theia specifications (see [17], for details) which target astrometric end-of-mission precisions of $10 \mu$ as for a faint object of $R=20 \mathrm{mag}$ and $0.15 \mu$ as for a bright object of $R=5 \mathrm{mag}$ (see Table 1 ).

\section{Science questions}

Europe has long been a pioneer of astrometry, from the time of ancient Greece to Tycho Brahe, Johannes Kepler, the Copernican revolution and Friedrich Bessel. ESA's Hipparcos [59, 103] and Gaia [42] satellites continued this tradition, revolutionizing our view of the Solar Neighborhood and Milky Way, and providing a crucial foundation for many disciplines of astronomy.

An unprecedented microarcsecond relative precision mission will advance European astrometry still further, setting the stage for breakthroughs on the most critical questions of cosmology, astronomy, and particle physics.

\subsection{Dark matter}

The current hypothesis of cold dark matter (CDM) urgently needs verification. Dark matter $(\mathrm{DM})$ is essential to the $\Lambda+\mathrm{CDM}$ cosmological model $(\Lambda \mathrm{CDM})$, which successfully describes the large-scale distribution of galaxies and the angular fluctuations of the Cosmic Microwave Background, as confirmed by ESA's Planck mission [104]. Dark matter is the dominant form of matter $(\sim 85 \%)$ in the Universe, and ensures the formation and stability of enmeshed galaxies and clusters of galaxies. The current paradigm is that dark matter is made of heavy, hence cold, particles, otherwise galaxies will not form. However, the nature of dark matter is still unknown.

There are a number of open issues regarding $\Lambda \mathrm{CDM}$ on small scales. Simulations based on DM-only predict 1) a large number of small objects orbiting the Milky Way, 2) a steep DM distribution in their centre and 3) a prolate Milky Way halo. However, hydrodynamical simulations, which include dissipative gas and powerful astrophysical phenomena (such as supernovae explosions and jets from galactic nuclei) can change this picture. Quantitative predictions are based on very poorly understood sub-grid physics and there is no consensus yet on the results. Answers are buried at small-scales, which are extremely difficult to probe. A new high precision astrometric mission appears to be the best way to settle the nature of DM and will allow us to validate or refute key predictions of $\Lambda \mathrm{CDM}$, such as

- the DM distribution in dwarf spheroidal galaxies

- the outer shape of the Milky Way DM halo

- the lowest masses of the Milky Way satellites and subhalos 
- $\quad$ the power spectrum of density perturbations

These observations will significantly advance research into DM. They may indicate that $\mathrm{DM}$ is warmer than $\triangle \mathrm{CDM}$ predicts. Or we may find that $\mathrm{DM}$ is prone to selfinteractions that reduces its density in the central part of the satellites of the Milky Way. We may discover that DM has small interactions that reduce the number of satellite companions. Alternatively, measurement of the Milky Way DM halo could reveal that DM is a sophisticated manifestation of a modification of Einstein's gravity.

\subsubsection{The DM distribution in dwarf spheroidal galaxies}

Because they are DM-dominated (see Fig. 1 where the number of stars versus the massto-light ratio is presented), dwarf Spheroidal galaxies (dSphs) are excellent laboratories to test the distribution of DM within the central part of small galaxies and disentangle the influence of complex baryonic processes from that of DM at these scales.

Simulations from [97] or [108] for example show that the DM distribution (referred to as DM profile) in dSphs strongly depends on their star formation history. More specifically, these simulations find that CDM can be heated by bursty star

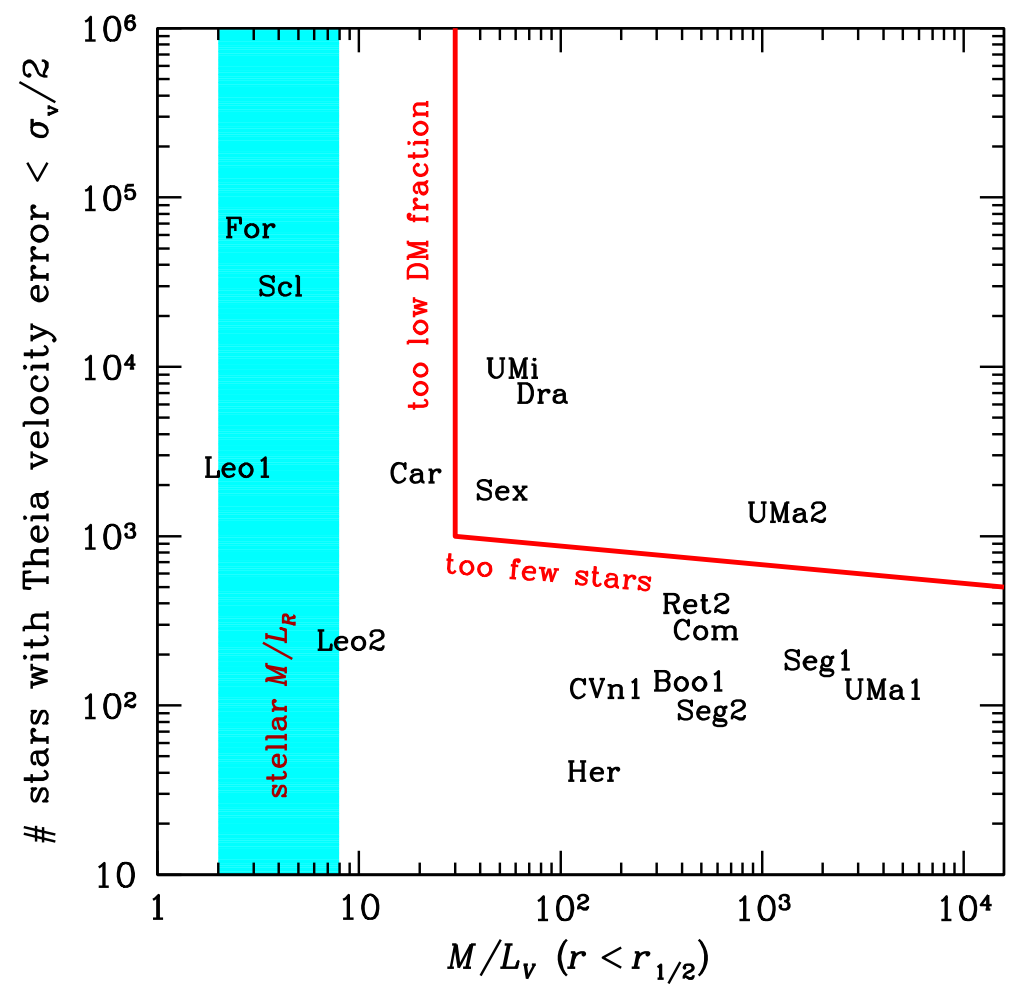

Fig. 1 Number of dwarf spheroidal galaxy stars within the field of view of Theia, a high precision astrometry concept, with expected plane-of-sky errors lower than half the galaxy's velocity dispersion as a function of the galaxy's estimated mass-to-light ratio within the effective (half-projected-light) radius of the galaxy. Luminosities and total masses within the half-light radii are mainly from [142] 
formation inside the stellar half-light radius $r_{1 / 2}$, if star formation proceeds for long enough. As a result, some dSphs like Fornax have formed stars for almost a Hubble time and so should have large central DM cores, while others, like Draco and Ursa Major 2, had their star formation truncated after just $\sim 1-2$ Gyrs and should retain their steep central DM cusp.

Large DM cores could also be attributed however to strong self-interactions. Hence finding evidence for such cores in the faintest dSphs (which are even more DM dominated [149] than the classical ones), will bring tremendous insights about the history of baryonic processes in these objects and could even dramatically change our understanding of the nature of DM. Indeed, self-interacting DM [129] is expected to scatter in the dense inner regions of dSphs, and thus leads to homogeneous cores. Finding such a core DM distribution in dSphs could then reveal a new type of particle forces in the DM sector and provide us with new directions to build extensions of the Standard Model of particle physics. On the other hand, finding cuspy DM profiles in all $\mathrm{dSphs}$ (including the faintest ones) will confirm $\Lambda \mathrm{CDM}$ and place strong constraints on galaxy formation. As shown in Figs. 16 and 17, a telescope with microarcsecond astrometric precision allows us to determine whether the DM distribution in $\mathrm{dSphs}$ is cuspy or has a core, and hence can lead to a very significant breakthrough regarding the nature of DM.

To determine the inner DM distribution in dSphs, one needs to remove the degeneracy between the radial DM profile and orbital anisotropy that quantifies whether stellar orbits are more radial or more tangential in the Jeans equation [15]. This can be done by adding the proper motions of stars in dSphs. Figure 2 shows that for the Draco dSph (which was obtained using single-component spherical mock datasets from the Gaia Challenge Spherical and Triaxial Systems working group, ${ }^{1}$ and the number of stars expected to be observed by a high precision astrometry mission), the inclusion of proper motions lifts the cusp / core degeneracy that line-of-sight-only kinematics cannot disentangle.

We remark in addition that a high precision astrometric mission is able to perform follow-ups of Gaia's observations of dSphs streams of stars if needed. Not only will such a mission provide the missing tangential velocities for stars with existing radial velocities, but it will also provide crucial membership information - and tangential velocities - for stars in the outer regions of the satellite galaxies that are tidally disrupted by the Milky Way.

\subsubsection{The triaxiality of the Milky Way dark matter halo}

For almost two decades cosmological simulations have shown that Milky Way-like DM halos have triaxial shapes, with the degree of triaxiality varying with radius ([36, 70], for example): halos are more round or oblate at the center, become triaxial at intermediate radii, and prolate at large radii [156].

Precise measurement of the velocity of distant Hyper Velocity Stars (hereafter HVS) can test these departures from spherical symmetry, independently of any other

\footnotetext{
${ }^{1}$ See http://astrowiki.ph.surrey.ac.uk/dokuwiki/doku.php?id=tests:sphtri
} 

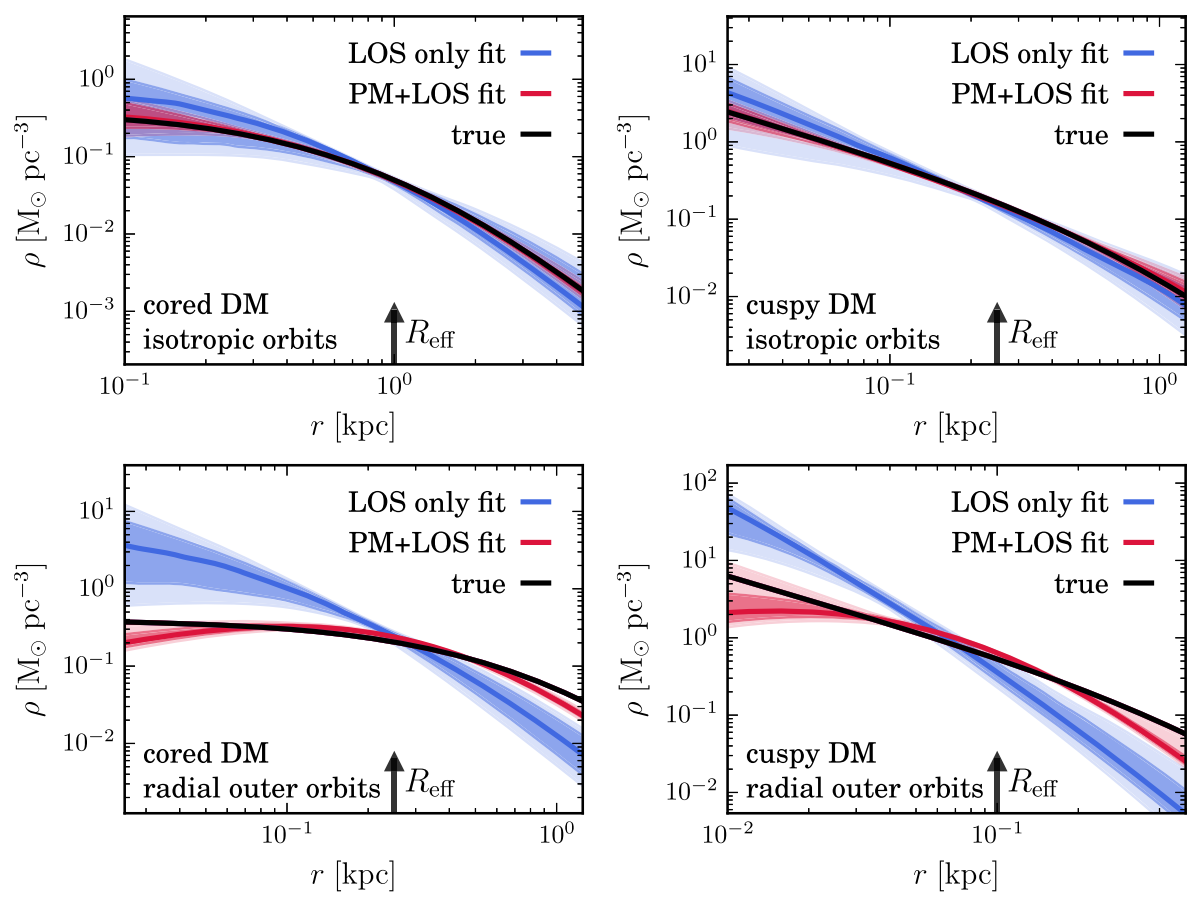

Fig. 2 Reconstruction of the DM halo profile of the Draco dSph without (blue) and with (red) proper motions using the mass-orbit modeling algorithm of [145]. Four mocks of Draco [143] were used, with cored (left) and cuspy (right) DM halos, and with isotropic velocities everywhere (top) or only in the inner regions with increasingly radial motions in the outer regions (bottom). The effective (half-projected light) radii of each mock is shown with the arrows. The stellar proper motions in the mocks were perturbed with apparent magnitude dependent errors as expected with 1000 hours of observations spread over 4 years

technique attempted so far (such as the tidal streams). HVSs were first discovered serendipitously $[25,37,56]$, and later discovered in a targeted survey of blue mainsequence stars ([23], and references therein). Gaia measurements demonstrate that candidate hyper velocity stars include unbound disc runaways [65], unbound white dwarfs ejected from double-degenerate type Ia supernovae [123], and runaways from the LMC [38], however the highest-velocity main sequence stars in the Milky Way halo have trajectories that point from the Galactic Center [26, 74].

Because these velocities exceed the plausible limit for a runaway star ejected from a binary, in which one component has undergone a supernova explosion, the primary mechanism for a star to obtain such an extreme velocity is assumed to be a threebody interaction and ejection from the deep potential well of the supermassive black hole at the Galactic Center [55, 154].

By measuring the three-dimensional velocity of these stars, we will reconstruct the triaxiality of the Galactic potential. In a spherical potential, unbound HVS ejected from the Galactic Center should travel in nearly a straight line, as depicted in Fig. 3. However, for triaxial halos, the present velocity vector should not point exactly 

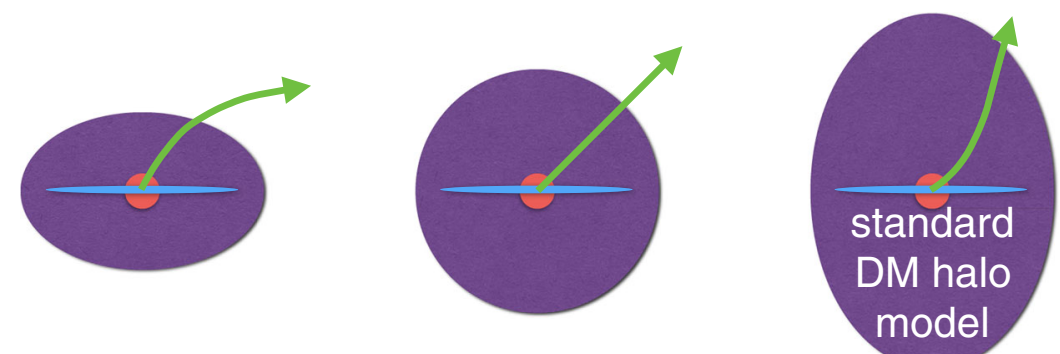

Fig. 3 Illustration of the trajectories of hyper velocity stars ejected from Galactic Center for 3 different outer DM halo shapes: oblate (left), spherical (middle), and prolate (right)

from the Galactic Center because of the small curvature of the orbit caused by nonspherically symmetric part of the potential [46, 153]. While both the halo and stellar disc induce transverse motions, the effect is dominated by halo triaxiality at the typical distance of HVSs. The deflection contributed by the disc peaks around $10 \mathrm{kpc}$ but quickly declines at larger distances, while the deflection due to the triaxial halo continues to accumulate along the whole trajectory. Figure 4 shows the spread of proper motion for one star, HVS5, for different halo shapes (different halo axis ratios and different orientations of the major axis).

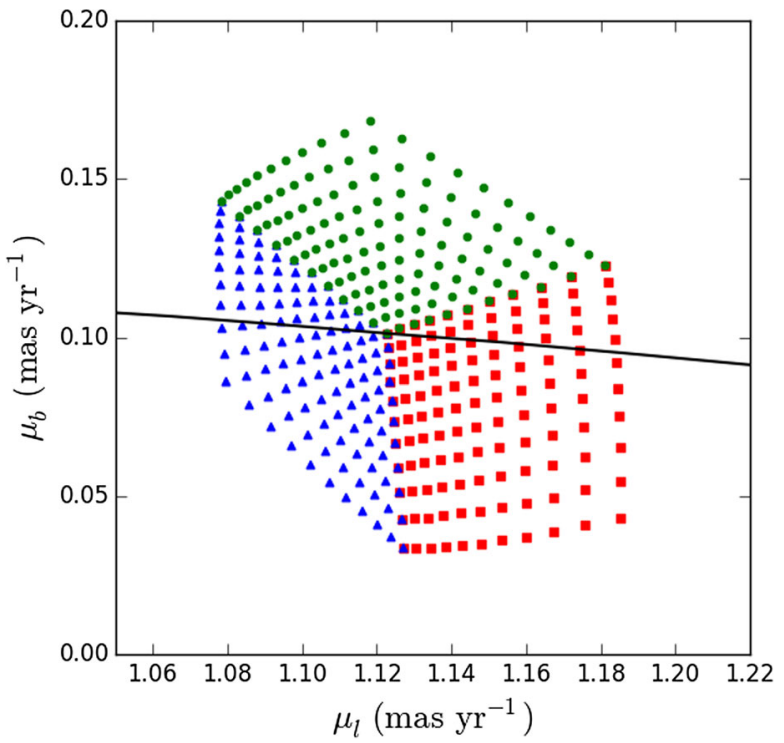

Fig. 4 Expected proper motions of HVS5 under different assumptions about the shape and orientation of the DM halo. The families of models are shown with the halo major axis along the Galactic X- (red squares), Y- (blue triangles), and Z- (green circles) coordinates. The solid line shows how the centroid of the proper motions will shift with a different distance to HVS5 
Proper motions of several HVSs were measured with the Hubble Space Telescope (HST) by [24], using an astrometric frame based on background galaxies. However, these measurements were not sufficiently accurate to constrain the halo shape or the origin of each HVS. A high precision astrometric mission with a sufficiently large field of view could include about 10 known quasars from the SDSS catalog around most HVSs. This will provide a much more stable and accurate astrometric frame, and will allow us to constrain the halo axis ratios to about $5 \%$.

Figure 5 shows that with a precision of $4 \mu$ as/yr one can constrain the orientation of the halo major axis and measure the axis ratios to an accuracy of $\delta\left(q_{Z} / q_{X}\right)<0.05$ for the typical HVS distance of $50 \mathrm{kpc}$. For comparison, Gaia at the end of its mission will achieve only $40-150 \mu \mathrm{as} / \mathrm{yr}$, which is insufficient to provide useful constraints on the axis ratios.

Statistical studies of high-precision proper motions of HVSs can also constrain departures of the halo shape from spherical ([43], in preparation). Indeed, numerical simulations of the trajectories of synthetic HVSs ejected through the Hills mechanism show that the distributions of the HVS tangential velocities in the Galactocentric reference frame are significantly different from spherical and non-spherical halos: the significance is $P \leq 1.3 \times 10^{-6}$ for oblate halos with $q_{Z} / q_{X} \leq 0.9$ and $P \leq$ $2.2 \times 10^{-5}$ for prolate halos with $q_{Z} / q_{X} \geq 1.1$. The median tangential velocity of a sample of $\sim 100 \mathrm{HVSs}$ located at heliocentric distances $\sim 50 \mathrm{kpc}$ can differ by

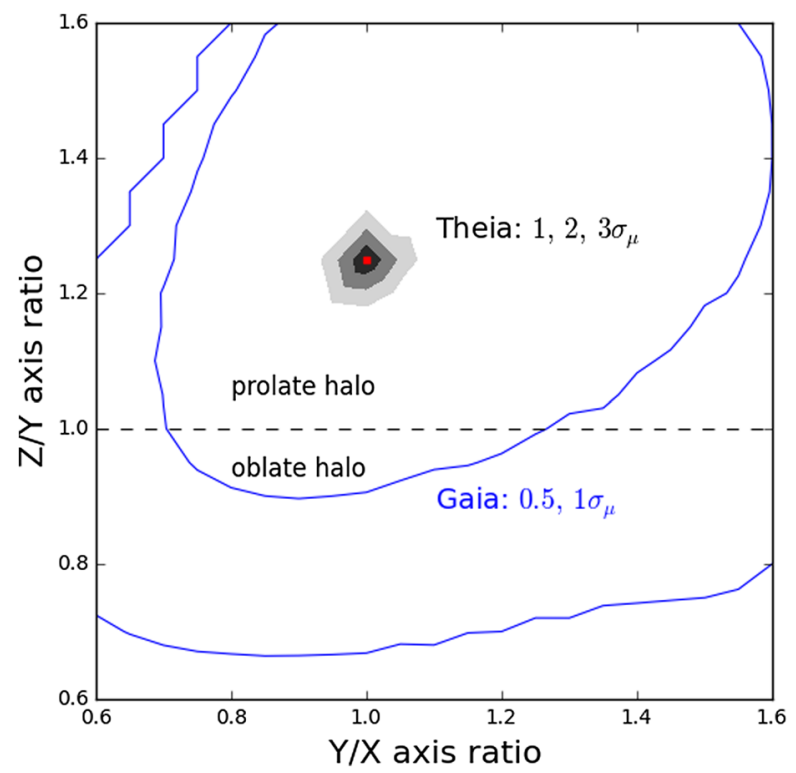

Fig. 5 Example of a reconstruction of the Galactic halo shape from a high precision astrometry mission (Theia) measurement of proper motion of HVS5. The assumed proper motions correspond to a prolate model with $q_{X}=q_{Y}=0.8 q_{Z}$, marked by a red square. Shaded contours represent confidence limits corresponding to the expected 1,2 , and $3 \sigma_{\mu}$ proper motion errors. The outer blue contours show the accuracy that will be achieved by Gaia at the end of its mission, even if its expected error was reduced by a factor of 2 
$\sim 5-10 \mathrm{~km} / \mathrm{s}$, implying differences in proper motions of $\sim 20-40 \mu \mathrm{as} / \mathrm{yr}$ between spherical and non-spherical halos.

Finally, an accurate measurement of HVS velocities may lead to improved understanding of the black hole(s) at the Galactic Center. Indeed, theoretical models show that HVSs will have a different spectrum of ejection velocities from a binary black hole versus a single massive black hole. Gaia has led to the discovery of several candidate hypervelocity stars (ejection velocities of over $550 \mathrm{~km} / \mathrm{s}:,[50,66,88]$ ), that were definitely not ejected from the Galactic Center but were ejected from spiral arms in the Milky Way disc. These possibly point to intermediate mass black holes of mass $100 \mathrm{M}_{\odot}$ - these could be local remnants of binary black hole mergers of the kind discovered by LIGO/Virgo [5, 6] and could have important implications for our understanding of stellar evolution.

\subsubsection{Orbital distribution of dark matter from the orbits of halo stars}

The orbits of DM particles in halos ${ }^{2}$ cannot be detected directly since DM particles interact only weakly with normal matter. However, in a triaxial potential such as described above, it is expected that a large fraction of the DM orbits do not have any net angular momentum. Hence these particles should get arbitrarily close to the center of the cusp, regardless of how far from the center they were originally. This allows DM particles, which annihilate within the cusp to be replenished on a timescale $10^{4}$ times shorter than in a spherical halo (analogous to loss cone filling in the case of binary black holes, see [91]).

Recent work on the orbital properties and kinematic distributions of halo stars and DM particles show that halo stars, especially the ones with lowest metallicities, are relatively good tracers of DM particles $[53,54,141]$ and observations with Gaia Data Release 2 (DR2) may have already led to the kinematic discovery of dark substructure [96]. The orbits reflect both the accretion/formation history and the current shape of the potential because DM halos are dynamically young (i.e. they are still growing and have not attained a long term equilibrium configuration where all orbits are fully phase mixed). This opens up the very exciting possibility that one can infer the kinematical distribution of DM particles by assuming that they are represented by the kinematics of halo stars.

\subsubsection{Perturbations by dark matter subhalos}

A central prediction of $\Lambda \mathrm{CDM}$ in contrast to many alternatives of DM, such as warm $\mathrm{DM}$ (e.g. [118]) or interacting DM (e.g. [18]), is the existence of numerous $10^{6}$ to $10^{8}$ $\mathrm{M}_{\odot}$ DM subhalos in the Milky Way halo. Their detection is extremely challenging, as they are very faint and lighter than dSphs. However, N-body simulations of the Galactic Disc show that such a DM halo passing through the Milky Way disc will warp the disc and produce a motion (bending mode), as shown in Fig. 6. This opens new avenues for detection as such perturbations of the disc will result in anomalous

\footnotetext{
${ }^{2}$ For an analysis of orbital content of DM halos see [27, 138-140].
} 


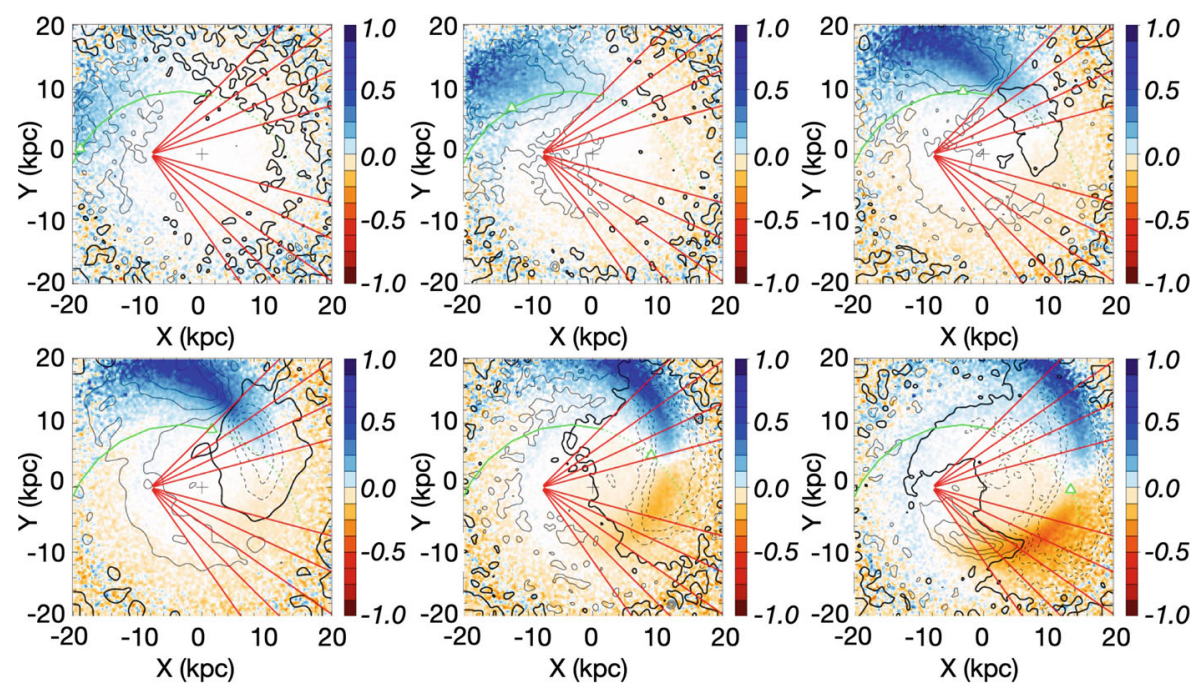

Fig. 6 Face-on view of the evolution of the perturbation of a Galactic Disc due to a DM subhalo of mass $3 \%$ of the mass of the disc crossing the disc from above. The upper and lower panels are before and after the crossing, respectively, for different times 125, 75 and $25 \mathrm{Myr}$ before the crossing and 25,75,125 Myr after (from left to right). The mean displacement amplitude is indicated in the color bar, while the contours indicate the amplitude of the bending mode in velocity space, using plain lines for positive values and dashed lines for negative values. The green line shows the projected orbit of the subhalo (dashed line after the impact with the disc). The green triangle shows the current location of the subhalo on its orbit. The red lines are our potential lines of sight for Theia, a high precision astrometry mission concept, spaced by $10^{\circ}$ in longitude with one pointing above the plane and one below the plane, that will allow us to map the disc perturbation behind the Galactic Center

motions of the stars in the disc (e.g. [39], for recent analysis), that could give rise to an astrometric signal.

These anomalous bulk motions develop both in the solar vicinity [147] and on larger scales [39], see Fig. 7. Therefore, measuring very small proper motions of individual faint stars in different directions towards the Galactic Disc could prove the existence of these subhalos and confirm the CDM scenario. Alternatively, in case they are not found, high precision astrometric observations will provide tantalizing evidence for alternative DM scenarios, the most popular today being a warmer form of DM particle, though these results could also indicate DM interactions [18].

A field of view of $1^{\circ} \times 1^{\circ}$ in the direction of the Galactic Disc has $\sim 10^{6}$ stars with an apparent magnitude of $R \leq 20$ (given by the confusion limit). Given the astrometric precisions per field of view of Fig. 17, a high precision astrometric instrument could detect up to 7 impacts on the disc from sub-halos as small as a few $10^{6} \mathrm{M}_{\odot}$.

Gaia DR2 astrometry has led to the discovery of gaps in tidal streams [105] like the GD1 stream. The gaps and off-stream stars (spur) are consistent with gravitational interactions with compact DM subhalos. Furthermore, Gaia DR2 data have revealed that globular cluster streams (GD1 and Jhelum) show evidence for cocoon-like structures that most likely arise from evolution inside a (dark) subhalo prior to their tidal disruption by the Milky Way itself $[19,28,88]$. The high astrometric precision of a 

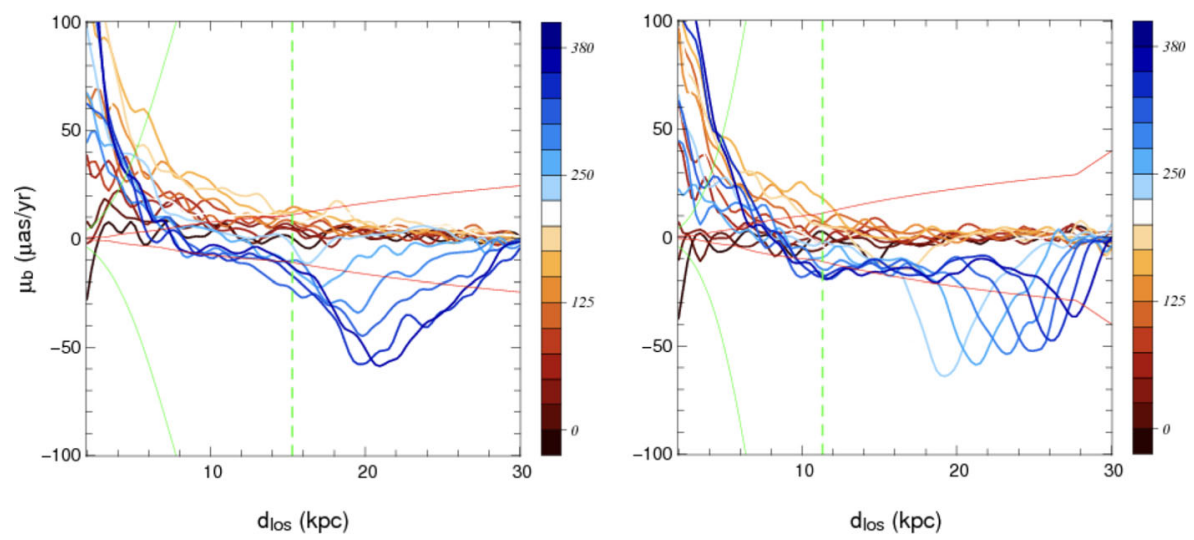

Fig. 7 Astrometric signatures in the proper motion along Galactic latitude of the perturbation of disc stars by a subhalo. The left and right panels show lines of sight as a function of distance along the line of sight and time, for $\ell=-25^{\circ}$ and $\ell=+25^{\circ}$ respectively for $b=+2^{\circ}$. The color codes the time in Myr, red for times prior to the crossing of the plane by the satellite, blue for later times. The green line is Gaia's expected end of mission performance for a population of red clump stars along these lines of sight. The vertical dashed line is Gaia's detection limit $(G=20)$ for the same population. The red lines are Theia's expected $1 \sigma$ accuracy for the same stars and for a $400 \mathrm{~h}$ exposure of the field over the course of the mission

Theia-like mission will enable us to measure the small velocity perturbations around the gaps in streams and allow for a much more accurate determination of both the masses and density structures of the perturbing dark subhalos.

\subsubsection{Ultra-compact minihalos of dark matter in the Milky Way}

In the $\Lambda \mathrm{CDM}$ model, galaxies and other large-scale structures formed from tiny fluctuations in the distribution of matter in the early Universe. Inflation predicts a spectrum of primordial fluctuations in the curvature of spacetime, which directly leads to the power spectrum of initial density fluctuations. This spectrum is observed on large scales in the cosmic microwave background and the large scale structure of galaxies, but is very poorly constrained on scales smaller than $2 \mathrm{Mpc}$. This severely restricts our ability to probe the physics of the early Universe. A high precision astrometric mission could provide a new window on these small scales by searching for astrometric microlensing events caused by ultra-compact minihalos (UCMHs) of DM.

UCMHs form shortly after matter domination (at $z \sim 1000$ ), in regions that are initially overdense (e.g. $\delta \rho / \rho>0.001$ in, [114]). UCMHs only form from fluctuations about a factor of 100 larger than their regular cosmological counterparts, so their discovery will indicate that the primordial power spectrum is not scale invariant. This will rule out the single-field models of inflation that have dominated the theoretical landscape for the past thirty years. Conversely, the absence of UCMHs can be used to establish upper bounds on the amplitude of the primordial power spectrum on small scales [22], which will rule out inflationary models that predict enhanced small-scale structure [13]. 
Like standard DM halos, UCMHs are too diffuse to be detected by regular photometric microlensing searches for MAssive Compact Halo Objects (MACHOs). Because they are far more compact than standard DM halos, they however produce much stronger astrometric microlensing signatures [81]. By searching for microlensing events due to UCMHs in the Milky Way, a high precision astrometric mission will provide a new probe of inflation. A search for astrometric signatures of UCMHs in the Gaia dataset could constrain the amplitude of the primordial power spectrum to be less than about $10^{-5}$ on scales around $2 \mathrm{kpc}$ [81]. Figure 8 shows that higher astrometric precision (corresponding to that of Fig. 17) will provide more than an order of magnitude higher sensitivity to UCMHs, and around four orders of magnitude greater mass coverage than Gaia. These projections are based on $8000 \mathrm{hr}$ of observations of 10 fields in the Milky Way disc, observed three times a year, assuming that the first year of data is reserved for calibrating stellar proper motions against which to look for lensing perturbations. Figure 9 shows that a high precision astrometric mission will test the primordial spectrum of perturbations down to scales as small as $700 \mathrm{pc}$, and improve on the expected limits from Gaia by over an order of magnitude at larger scales.

The results will be independent of the DM nature, as astrometric microlensing depends on gravity only, unlike other constraints at similar scales based on DM annihilation, from the Fermi Gamma-Ray Space Telescope [22]. An astrometric mission with higher precision (shown in Fig. 17) will have sensitivity four orders of magnitude better than constraints from the absence of primordial black holes (PBHs; , [68]),

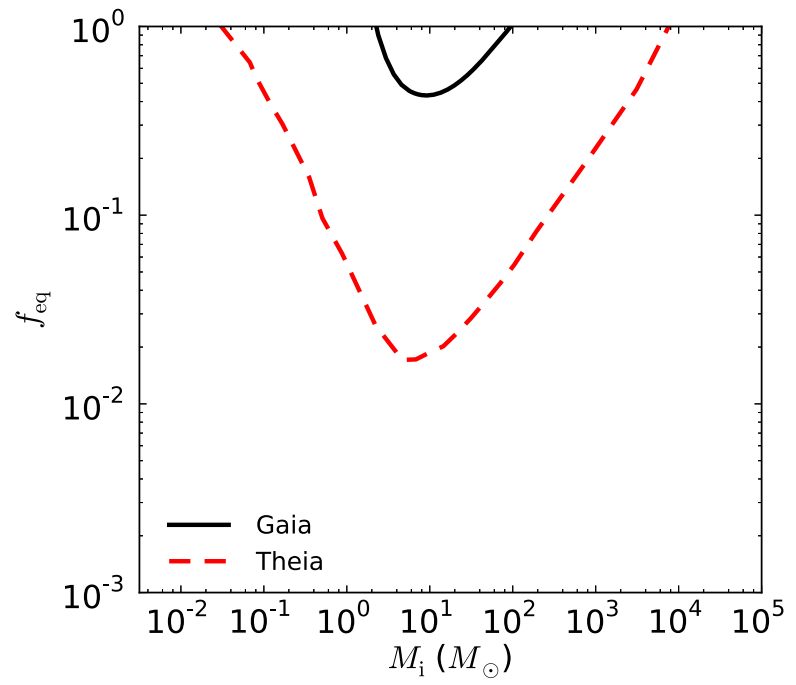

Fig. 8 Projected sensitivity of a high precision astrometry mission (Theia) to the fraction of dark matter in the form of ultra-compact minihalos (UCMHs) of mass $M_{i}$ at the time of matter-radiation equality. Smaller masses probe smaller scales, which correspond to earlier formation times (and therefore to later stages of inflation). A UCMH mass of $0.1 \mathrm{M}_{\odot}$ corresponds to a scale of just $700 \mathrm{pc}$. Expected constraints from Gaia are given for comparison, showing that a Theia-like mission will provide much stronger sensitivity, as well as probe smaller scales and earlier formation times than ever reached before 


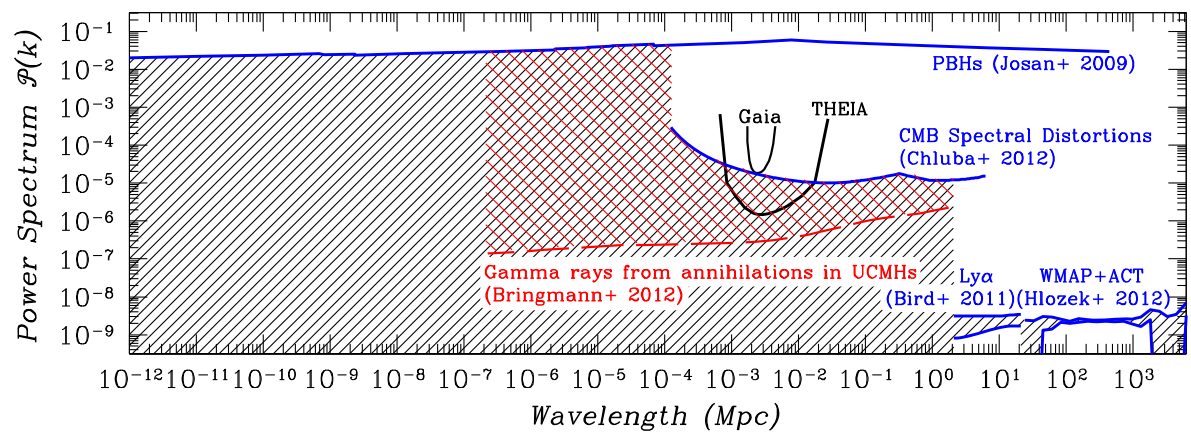

Fig. 9 Limits on the power of primordial cosmological perturbations at all scales, from a range of different sources. A Theia-like mission will provide far stronger sensitivity to primordial fluctuations on small scales than Gaia, spectral distortions or primordial black holes (PBHs). Unlike gamma-ray UCMH limits, a high precision astrometry mission's sensitivity to cosmological perturbations will also be independent of the specific particle nature of dark matter

and more than an order of magnitude better than CMB spectral distortions [29], which give the current best model-independent limit on the primordial power spectrum at similar scales.

\subsubsection{Directly testing gravity}

Using the nearest star, Proxima Centauri, astrometry could measure the behaviour of gravity at low accelerations. A high precision astrometry mission with an extended baseline of 10 years and a precision of $0.5 \mu$ as could measure the wide binary orbit of Proxima Centauri around Alpha Centauri A and B to distinguish between Newtonian gravity and Milgromian dynamics (MOND). The separation between Proxima Centauri and the Alpha Centauri system suggests orbital acceleration that is significantly less than the MOND acceleration constant $a_{0} \sim 1.2 \times 10^{-10} \mathrm{~m} / \mathrm{s}^{2}$ [14]. It would be the first direct measurement of the departure from Newtonian gravity in the very weak field limit, as expected in MOND, and the results could have profound implications for fundamental physics.

\subsection{Exoplanets}

\subsubsection{The frontier of exoplanet astrophysics}

The ultimate exoplanetary science goal is to answer the enigmatic and ancient question, "Are we alone?" via unambiguous detection of biogenic gases and molecules in the atmosphere of an Earth twin around a Sun-like star [121]. Directly addressing this age-old question related to the uniqueness of the Earth as a habitat for complex biology constitutes today the vanguard of the field, and it is clearly recognized as an unprecedented, cross-technique, interdisciplinary endeavor.

Since the discovery of the first Jupiter-mass companion to a solar-type star [90], tremendous progress has been made in the field of exoplanets. Our knowledge is 
expanding quickly due to the discovery of thousands of planets, and the skillful combination of high-sensitivity space-borne and ground-based programs that have unveiled the variety of planetary systems architectures that exist in the Galaxy (e.g. $[63,89])$. Preliminary estimates (e.g. [148]) are now also available for the occurrence rate $\eta_{\uplus}$ of terrestrial-type planets in the Habitable Zone (HZ) of stars more like the Sun $\left(\eta_{\uplus} \sim 10 \%\right)$ and low-mass M dwarfs $\left(\eta_{\uplus} \sim 50 \%\right)$.

However, transiting or Doppler-detected HZ terrestrial planet candidates (including the discovery of the $m_{\mathrm{p}} \sin i=1.3 M_{\oplus} \mathrm{HZ}$-planet orbiting Proxima Centauri; [9]) lack determinations of their bulk densities $\varrho_{\mathrm{p}}$. Thus, the HZ terrestrial planets known to date are not amenable to making clear statements on their habitability. The K2 [64], TESS [113], and PLATO [107] missions are bound to provide tens of Earths and Super-Earths in the $\mathrm{HZ}$ around bright $\mathrm{M}$ dwarfs and solar-type stars for which the estimates of their bulk densities $\varrho_{\mathrm{p}}$ might be obtained in principle, but atmospheric characterization for the latter sample might be beyond the capabilities of JWST and the ground-based telescopes with very large aperture diameters. The nearest stars to the Sun are thus the most natural reservoir for the identification of potentially habitable rocky planets that might be characterized via a combination of high-dispersion spectroscopy and high-contrast imaging with the ground-based telescopes [126] or via coronagraphic or interferometric observations in space [80].

Unlike the Doppler and transit methods, astrometry alone can determine reliably and precisely the true mass and three-dimensional orbital geometry of an exoplanet, which are fundamental inputs to models of planetary evolution, biosignature identification, and habitability. By determining the times, angular separation and position angle at periastron and apoastron passage, exquisitely precise astrometric position measurements will allow the prediction of where and when a planet will be at its brightest (and even the likelihood of a transit event), thus (a) crucially helping in the optimization of direct imaging observations and (b) relaxing important model degeneracies in predictions of the planetary phase function in terms of orbit geometry, companion mass, system age, orbital phase, cloud cover, scattering mechanisms, and degree of polarization (e.g. [85]). Only a high precision astrometric mission's observations will have the potential to 1) discover most of the potentially habitable planets around the nearest stars to the Sun, 2) directly measure their masses and system architectures, and 3) provide the most complete target list and vastly improve the efficiency of detection of potential habitats for complex exo-life with the next generation of space telescopes and ground-based very large aperture telescopes.

\subsubsection{Fundamental program}

Surgical single-point positional precision measurements in pointed, differential astrometric mode ( $<1 \mu \mathrm{as}$ ), could exploit a high precision astrometric mission's unique capability to search for the nearest Earth-like planets to the Sun. The amplitude $\alpha$ of the astrometric motion of a star due to an orbiting planet is (in microarcseconds):

$$
\alpha=3\left(\frac{M_{\mathrm{p}}}{M_{\oplus}}\right)\left(\frac{a_{\mathrm{p}}}{1 \mathrm{AU}}\right)\left(\frac{M_{\star}}{M_{\odot}}\right)^{-1}\left(\frac{D}{1 \mathrm{pc}}\right)^{-1} \mu \mathrm{as}
$$


where $M_{\star}$ is the stellar mass, $M_{\mathrm{p}}$ is the mass of the planet, $a_{\mathrm{p}}$ is the semi-major axis of the orbit of the planet, and $D$ is the distance to the star. For a terrestrial planet in the $\mathrm{HZ}$ of a nearby sun-like star, a typical value is $0.3 \mu$ as (an Earth at $1.0 \mathrm{AU}$ of a Sun, at $10 \mathrm{pc}$ ). This very small motion (the size of a coin thickness on the Moon as measured from the Earth) will be accessible to a high precision astrometric instrument by measuring the differential motion of the star with respect to far-away reference sources.

A core exoplanet program could be comprised of 63 of the nearest A, F, G, K, and $M$ stars (Fig. 10). Many of them are found in binary and multiple systems. Binary stars are compelling for a high precision astrometry space mission for a number of reasons. They are easier targets than single stars.

Furthermore, as the photon noise from the reference stars is the dominant factor of the error budget, the accuracy for binaries increases faster with telescope staring time than around single stars. For binaries, the reference stars only need to provide the plate scale and the reference direction of the local frame, the origin point coordinates

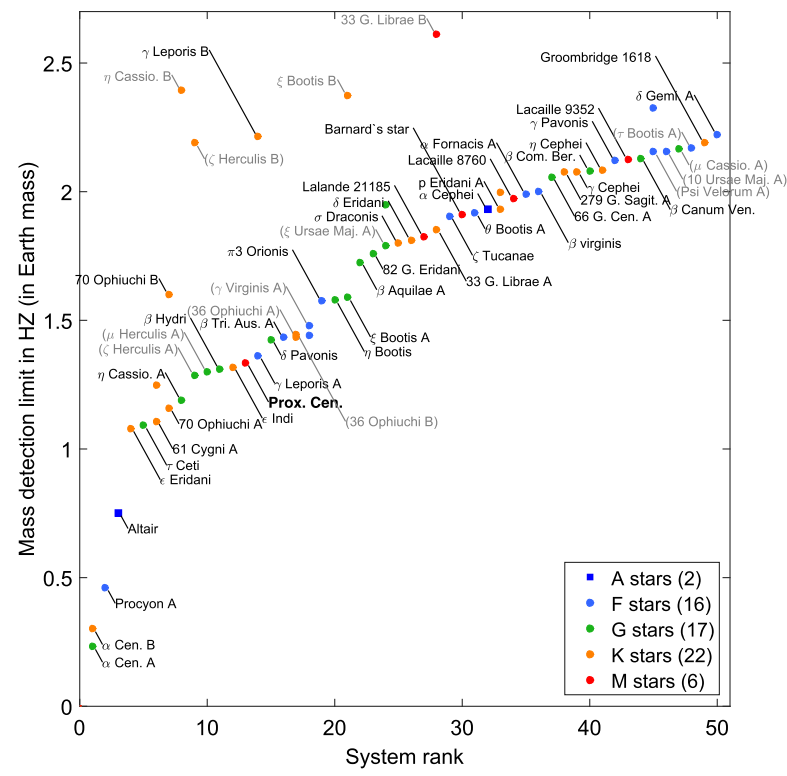

Fig. 10 Minimum masses of planets that can be detected at the center of the HZ of their star for the 63 best nearby A, F, G, K, M target systems. The target systems (either single or binary stars), are ranked from left to right with increasing minimum detectable mass in $\mathrm{HZ}$ around the primary system component, assuming equal observing time per system. Thus for binary stars, A and B components are aligned vertically, as they belong to the same system therefore they share the same rank. When the A and B mass thresholds are close the name is usually not explicitly written down to avoid overcrowding. B components that have mass thresholds above 2.2 $M_{\oplus}$ are named in gray and binaries that are estimated too close for followup spectroscopy are named in gray and in parenthesis. These binaries are expected to be only part of the secondary science program (planet formation around binaries). The star sample that is best for astrometry is similar to that of the best stars for spectroscopy in the visible, or in thermal IR (see text for explanations). Earths and Super-Earths with $M_{\mathrm{p}} \geq 1.5 M_{\oplus}$ can be detected and characterized (actual mass and full orbit) around 22 stars. All Super-Earths with $M_{\mathrm{p}}<2.2 M_{\oplus}$ can be detected and characterized around 59 stars 
are constrained by the secondary/primary component of the binary. Finally, when observing a binary, the astrometry on both components is obtained simultaneously: the staring time is only spent once as both components are within the same field of view (FoV). These two effects combined cause the observation of stars in binary systems to be much more efficient (as measured in $\mu$ as $\times \mathrm{h}^{-1 / 2}$ ) than that of single stars.

We further stress that the complete census of small and nearby planets around solar-type stars is unique to high-precision astrometry. On the one hand, Sun-like stars have typical activity levels producing Doppler noise of $\sim 1 \mathrm{~m} / \mathrm{s}$ (or larger), which is still 10 times the signal expected from an Earth-analog [82]. High precision space astrometry will be almost insensitive to the disturbances (spots, plages) due to stellar activity, having typical activity-induced astrometric signals with amplitude below $0.1 \mu$ as [76].

For the full sample of the nearest stars considered in Fig. 10 we achieve sensitivity (at the $6 \sigma$ level) to planets with $M_{p} \leq 3 M_{\oplus}$. If we consider $\eta_{\uplus} \sim 10 \%$, for the sample of 63 stars closest to our Solar System we thus expect to detect $\sim 6 \mathrm{HZ}$ terrestrial planets. Of these, 5 will be amenable for further spectroscopic characterization of their atmospheres ${ }^{3}$. A high precision astrometry mission could perform the measurements of the relevant stars and make a thorough census (95\% completeness) of these planets by using less than $10 \%$ of a four-year mission. As indicated above, this program will also be valuable for understanding planetary diversity, the architecture of planetary systems (2-d information plus Kepler's laws, results in 3-d knowledge) including the mutual inclination of the orbits, a piece of information that is often missing in our exploration of planetary systems.

\subsubsection{Additional exoplanet investigations}

A secondary program can help elucidate other important questions in exoplanetary science.

1. Planetary systems in S-Type binary systems. A high precision astrometry mission's performance for exoplanet detection around nearby binaries will be of crucial importance in revealing planet formation in stellar systems, the environment in which roughly half of main-sequence stars are born. The discovery of giant planets in binaries has sparked a string of theoretical studies, aimed at understanding how planets can form and evolve in highly perturbed environments [134]. Giant planets around one component of a binary (S-type orbits) have often been found in orbits very close to theoretical stability limits (e.g. [48, $117,133]$ ), and as for most of the binary targets the HZ of each component is stable, finding other and smaller bodies in their HZs is a real possibility. The contribution of a high precision astrometric mission could be decisive for these ongoing studies, by allowing the exploration of a crucial range of exoplanetary architectures in binaries.

\footnotetext{
${ }^{3}$ One target is a binary which is too close for follow-up spectroscopy
} 
2. Follow-up of known Doppler systems. Another unique use of a high precision astrometry mission will be the study of non-transiting, low-mass multiple-planet systems that have already been detected with Radial Velocities (RV). High precision astrometry will confirm or refute controversial detections, remove the $\sin i$ ambiguity and measure actual planetary masses. Furthermore, it will directly determine mutual inclination angles, which are critical to study (i) the habitability of exoplanets in multiple systems, since they modify the orientation of the spin axes and hence the way the climates change across time (e.g. [11, 21, 77]) and (ii) the dynamical evolution history of multiple systems, as e.g. coplanar orbits are indicative of smooth evolution, while large mutual inclinations and eccentricities point toward episodes of strong interactions, such as planet-planet scattering. Figure 11 illustrates a case where degeneracy in RV can be removed by astrometry. Using the proper motion difference technique or diagnostics representing 'excess' residuals to a single-star fit, there are a few Gaia-based results worth mentioning, such as mass constraints on the cool Super-Earth orbiting Proxima Centauri [71], the inferred true mass for HD 114762b [72], and the first high-quality measurement of highly mutually inclined orbits in the Pi Mensae system $[32,152]$.

3. Planetary systems on and off the main sequence. Gaia will be able to detect thousands of giant planetary companions around stars of all ages (including preand post-main-sequence), spectral type, chemical abundance, and multiplicity with results expected in the DR4 and DR5 data releases [106, 122, 128]. A high precision astrometry mission could cherry-pick from Gaia discoveries and identify systems amenable to follow-up to search for additional low-mass components in such systems, particularly in the regime of stellar parameters difficult for radial velocity work like early spectral types, young ages, very low metallicity, white dwarfs. Some of the systems selected might also contain transiting companions identified by TESS and PLATO (and possibly even Gaia itself), or planets directly imaged by SPHERE on the VLT or European Extremely Large Telescope.
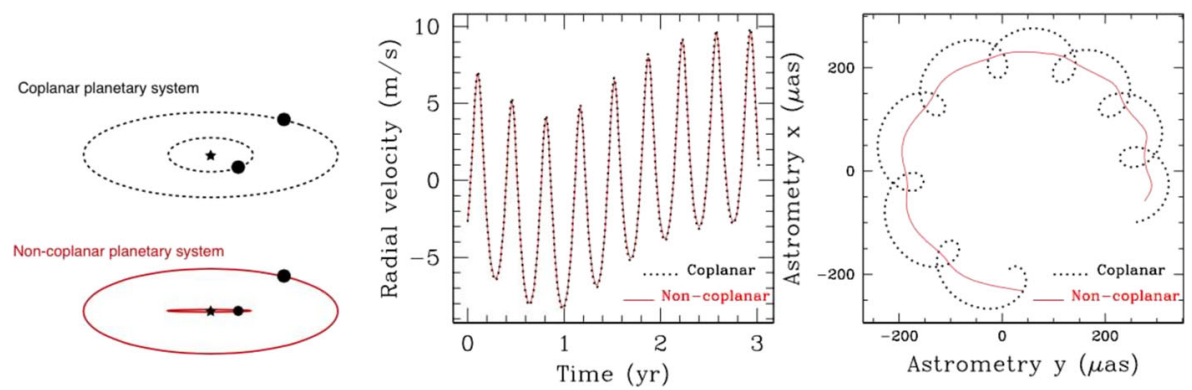

Fig. 11 An example where astrometry breaks the degeneracy. Two simulated planetary systems are around a solar-type star at $10 \mathrm{pc}$, with two Jupiter-like planets at 0.5 and $2.5 \mathrm{AU}$ (left). One is co-planar (dotted black line), the other has a mutual inclination of $30^{\circ}$ (full red line). The two corresponding RV curves are shown (middle), as well as the two astrometric ones (right). Curves are identical in the former case, but clearly separated in the latter revealing the inclined orbits 
4. Terrestrial planets around Brown Dwarfs. So far, among the few planetary mass objects that have been associated with brown dwarf (BD) hosts using direct imaging and microlensing techniques, only one is likely to be a low-mass planet ([137], and references therein)). However, there are both observational [111, $112,120]$ as well as theoretical $[92,102]$ reasons to believe that such systems could also be frequent around BDs. The recent identification of a trio of shortperiod Earth-size planets transiting a nearby star with a mass only $\sim 10 \%$ more massive than the hydrogen-burning limit [45] is a tantalizing element in this direction. In its all-sky survey, Gaia will observe thousands of ultra-cool dwarfs in the backyard of the Sun with sufficient astrometric precision to reveal any orbiting companions with masses as low as that of Jupiter [127]. A high precision astrometry mission could push detection limits of companions down to terrestrial mass. If the occurrence rate of $P \leq 1.3 \mathrm{~d}$, Earth-sized planets around BDs is $\eta=27 \%$ as suggested by [51] based on extrapolations from transit detections around late $\mathrm{M}$ dwarfs, the high precision measurements, probing for the first time a much larger range of separations with respect to transit surveys with sensitivity to low-mass planets, will unveil a potentially large number of such companions, and place the very first upper limits on their occurrence rates in case of null detection.

5. Astrometric effect of disks. As pointed out by [75], the photocenter of a star+disc system will have an elliptic motion for asymmetric discs. The latter is likely to mimic a planet or to perburb the characteriscs of an existing planet. Additional measurements (e.g. infrared flux) will be necessary to disentangle the disk asymmetry from a real planet.

\subsection{Compact objects}

\subsubsection{X-ray binaries}

The brightest Galactic X-ray sources are accreting compact objects in binary systems. Precise optical astrometry of these X-ray binaries provides a unique opportunity to obtain quantities which are very difficult to obtain otherwise. In particular, it is possible to determine the distances to the systems via parallax measurements and the masses of the compact objects by detecting orbital motion to measure the binary inclination and the mass function. With a high precision astrometric mission, distance measurements are feasible for $>50 \mathrm{X}$-ray binaries ${ }^{4}$, and orbital measurements will be obtained for dozens of systems. This will revolutionize the studies of X-ray binaries in several ways: here we discuss goals for neutron stars (NSs), including constraining their equation of state (EoS), and for black holes (BHs).

Matter in the NS interior is compressed to densities exceeding those in the center of atomic nuclei, opening the possibility to probe the nature of the strong interaction under conditions dramatically different from those in terrestrial experiments and to determine the NS composition. NSs might be composed of nucleons only; strange

\footnotetext{
${ }^{4}$ with 2000 hours of observation
} 
baryons (hyperons) or mesons might appear in the core or even deconfined quark matter, forming then a hybrid star with a quark matter core and hadronic matter outer layers; or of pure strange quark matter (a quark star). A sketch of the different possibilities is given in Fig. 12. Via the equation of state (EoS), matter properties determine the star's radius for a given mass. In particular, since general relativity limits the mass for a given EoS, the observation of a massive NS can exclude EoS models. Presently, the main constraint stems from the measurements of very massive NSs in radio pulsar/ white dwarf systems which have been reported with high precision [10, 12, 30, 35].

The key to constraining the NS EoS is to measure the masses and radii of NSs. While masses have been measured for a number of X-ray binary and radio pulsar binary systems (e.g., [79, 99]), the errors on the mass measurements for most X-ray binaries are large (see Fig. 13, left). The ultimate constraint on the EoS will be a determination of radius and mass of the same object, and a small number of such objects might be sufficient to pin down the entire EoS (e.g. [100]), see Fig. 13 (right), where several $M-R$ relations for different EoSs are shown. Current techniques to determine radii rely on spectroscopic measurements of accreting neutron stars, either in quiescence [52] or during thermonuclear (type I) X-ray bursts [99], and also timing observations of surface inhomogeneities of rotating NSs [49, 94].

A high precision astrometric mission will contribute by obtaining precise mass constraints with orbital measurements [135] and by improving distance measurements. Distances must be known accurately to determine the NS radii. For that purpose, new high precision data can be combined with existing and future X-ray data, e.g., from Athena, which is scheduled as the second large-class (L2) mission in ESA's Cosmic Vision. The Athena Science Working Group on the endpoints of

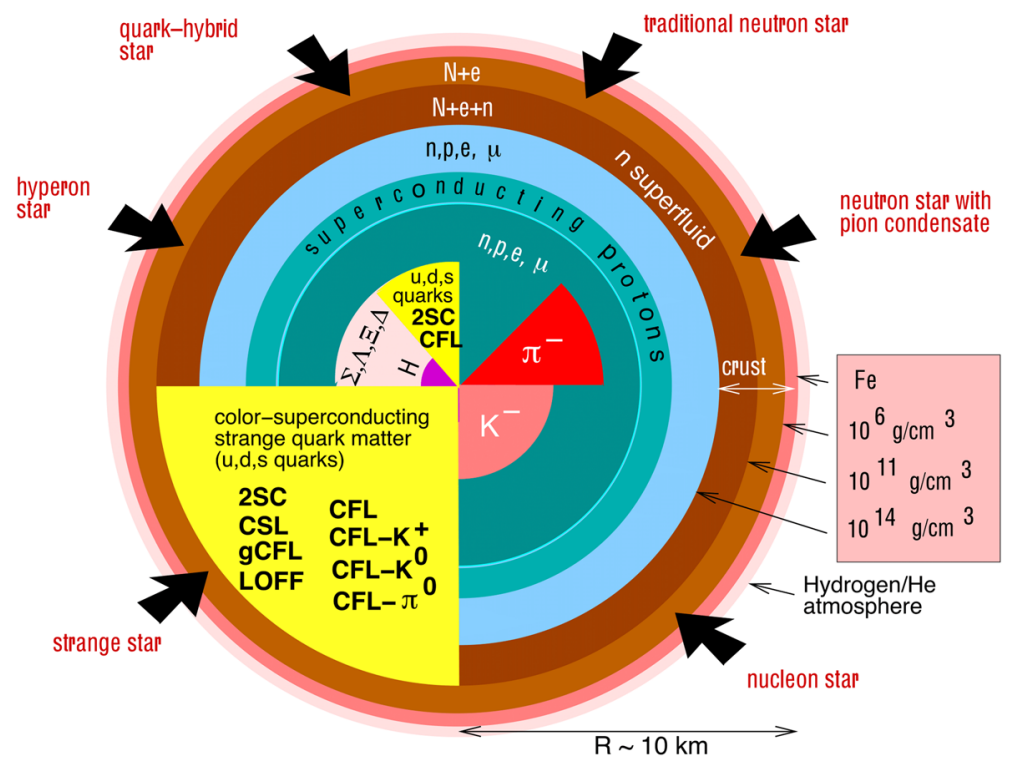

Fig. 12 Sketch of the different existing possibilities for the internal structure of a neutron star. Figure courtesy of [146] 

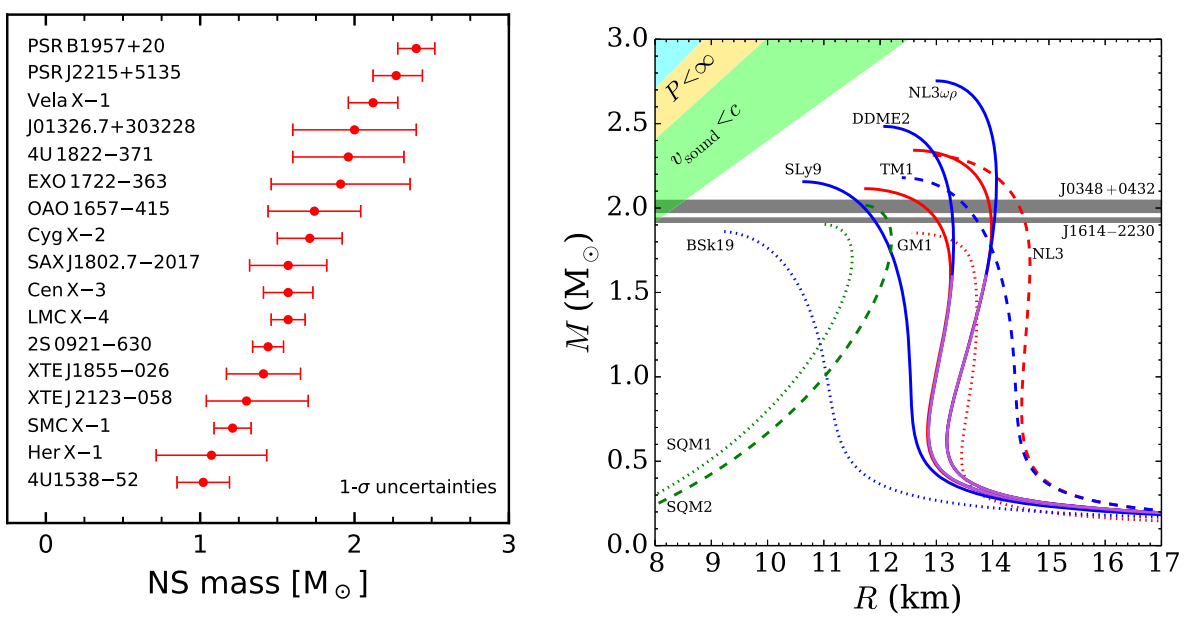

Fig. 13 Left: Neutron star mass measurements in X-ray binaries, update from [78], http://stellarcollapse. org. Right: $M-R$ relation for different EoS models (adapted from [41]): NS with a purely nucleonic core (in blue), with a core containing hyperons at high density (in red), and pure strange quark stars (in green). The horizontal grey bars indicate the masses of PSR J1614-2230 and PSR J0348+0432. The models indicated by dotted or dashed lines are either not compatible with NS masses or nuclear physics constraints. Note that a transition to matter containing hyperons is not excluded by present constraints

stellar evolution has observations of quiescent neutron star X-ray binaries to determine the NS EoS as its first science goal; however, their target list is restricted to systems that are in globular clusters. A high precision astrometric mission will enable distance measurements for many more NS X-ray binaries, allowing Athena to expand their target list.

Other techniques for constraining the NS EoS might also be possible in the future: detecting redshifted absorption lines; determining the NS moment of inertia of systems like the double pulsar J0737-3039; and more detections of tidal deformability from gravitational wave emission during the inspiral of a binary neutron star merger like for GW170817 [4]. GWs from the post-merger phase could strongly constrain the EoS, too. However, the mass and distance measurements that a high precision astrometric mission will obtain use techniques that are already well-established, providing the most certain opportunity for greatly increasing the numbers of NSs with mass or radius determinations.

In addition to the goal of constraining the NS EoS, NS masses are also relevant to NS formation and binary evolution. Current evolutionary scenarios predict that the amount of matter accreted, even during long-lived X-ray binary phases, is small compared to the NS mass. This means that the NS mass distribution is mainly determined by birth masses. Determining the masses of NSs in X-ray binaries, therefore, also provides a test of current accretion models and evolutionary scenarios, including the creation of the NSs in supernovae.

BHs are, according to the theory of general relativity, remarkably simple objects. They are fully described by just two parameters, their mass and their spin. Precise masses are available for very few BHs in X-ray binaries. The recent detection of gravitational waves [1] found in the binary BH mergers [2, 3] show that they have, 
on average, higher masses and probably lower spins than the BHs in X-ray binaries. These measurements are difficult to explain based on our understanding of stellar evolution and the fate of massive stars. Although BHs leave few clues about their origin, one more parameter that can be determined is the proper motion of BHs in $\mathrm{X}$-ray binaries. Measurements of proper motions provides information about their birthplaces and formation. It includes whether they were produced in a supernova (or hypernova) or whether it is possible for massive stars to collapse directly to BHs. A few BH X-ray binaries have proper motion measurements (e.g. [95]), but this number will rise dramatically with the astrometry measurements that a high precision astrometry mission will provide.

Currently, the cutting edge of research in BH X-ray binaries involves constraining $\mathrm{BH}$ spins, including the rate of spin and the orientation of the spin axis. Techniques for determining the rate of spin include measuring the relativistic broadening of the fluorescent iron $K_{\alpha}$ line in the X-ray emission and the study of the thermal continuum X-ray spectra [93, 110]. Concerning the direction of their spin axes, there is evidence that the standard assumption of alignment between the BH spin and orbital angular momentum axes is incorrect in some, if not many, cases [84, 136, 144], likely requiring a warped accretion disc. Theoretical studies show that such misalignments should be common [73]. However, binary inclination measurements rely on modeling the ellipsoidal modulations seen in the optical light curves [98], which is subject to systematic uncertainties, and a high precision astrometry mission will be able to provide direct measurements of orbital inclination for many of the BH X-ray binaries that show evidence for misalignments and warped discs.

\subsubsection{Astrometric microlensing}

In 1986 Bohdan Paczyński [101] proposed a new method for finding compact dark objects, via photometric gravitational microlensing. This technique relies on continuous monitoring of millions of stars in order to spot the temporal brightening due to space-time curvature caused by the presence and motion of a dark massive object. Microlensing reveals itself also in astrometry, since the centre of light of both unresolved images (separated by $\sim 1$ mas) changes its position while the relative brightness of the images changes in the course of the event. Astrometric time-series at sub-mas precision over the course of a couple of years will provide measurement of the size of the Einstein Ring, which combined with the photometric light curve, will directly yield the lens distance and mass. Most microlensing events are detected by large-scale surveys, e.g., OGLE and, in future possibly also the Rubin Observatory (previously known as the LSST). At typical brightness of V=19-20mag only a high-precision astrometry mission will be capable of providing good-enough astrometric follow-up of photometrically detected microlensing events (Fig. 14). Among 2000 events found every year, at least a couple should have a black hole as the lens, for which the mass measurement via astrometric microlensing will be possible.

Detection of isolated black holes and a complete census of masses of stellar remnants will for the first time allow for a robust verification of theoretical predictions of stellar evolution. Additionally, it will yield a mass distribution of lensing stars as well as hosts of planets detected via microlensing. 

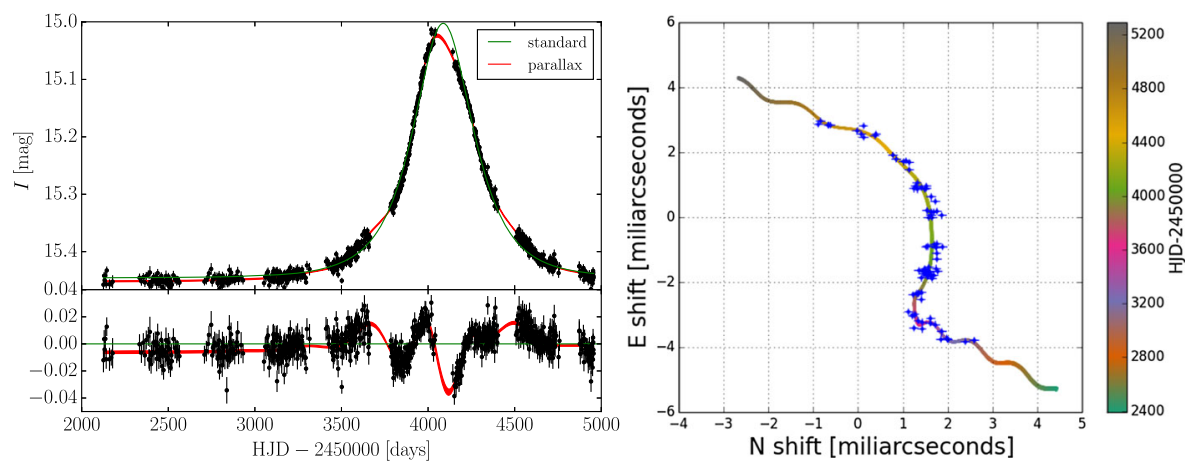

Fig. 14 Microlensing event, OGLE3-ULENS-PAR-02, the best candidate for a $\sim 10 \mathrm{M}_{\odot}$ single black hole. Left: photometric data from OGLE-III survey from 2001-2008. Parallax model alone can only provide mass measurement accuracy of 50-100\%. Right: simulated astrometric microlensing path for a similar event if observed with Theia, a high-precision astrometry mission. Combining this mission's superb astrometric accuracy with long-term photometric data will yield mass measurements of black holes and other dark compact object to $1 \%$ even at faint magnitudes

\subsection{Cosmic distance ladder}

The measurement of cosmological distances has revolutionized modern cosmology and will continue to be a major pathway to explore the physics of the early Universe. The age of the Universe $\left(H_{0}^{-1}\right)$ is a key measurement in non-standard DM scenarios. Its exact value is currently strongly debated, with a number of scientific papers pointing at discrepancies between measurement methods at the 2-3 $\sigma$ level. But the most serious tension with a discrepancy at the 3-4 $\sigma$ level appears between CMB estimates $\left(H_{0}=67.8 \pm 0.9 \mathrm{~km} / \mathrm{s} / \mathrm{Mpc}\right)$ or for that matter $\mathrm{BAO}$ results from the SDSS-III DR12 data combined with SNIa which indicate $H_{0}=67.3 \pm 1.0 \mathrm{~km} / \mathrm{s} /$ Mpc (see [8]) and measurements based on Cepheids and SNIa [115] giving $H_{0}=$ $73.24 \pm 1.74 \mathrm{~km} / \mathrm{s} / \mathrm{Mpc}$.

The tension between the methods can be due to unknown sources of systematics, to degeneracies between cosmological parameters, or to new physics (e.g. [69]). It is therefore of crucial importance to consider methods capable of measuring $H_{0}$ with no or little sensitivity to other cosmological parameters. Uncertainties can be drastically reduced by measuring time delays (TD) in gravitationally lensed quasars [109], as this technique only relies on well-known physics that is General Relativity. With enough statistics, and good modeling of the mass distribution in the lensing galaxy, TD measurements can lead to percent-level accuracy on $H_{0}$, independently of any other cosmological probe (e.g. , [20, 130, 132]). In practice, TDs can be measured by following the photometric variations in the images of lensed quasars. As the optical paths to the quasar images have different lengths and they intersect the lens plane at different impact parameters, the wavefronts along each of these paths reach the observer at different times. Hence the notion of TD.

Significant improvements in lens modeling, combined with long-term lens monitoring, should allow measuring $H_{0}$ at the percent level. The HOLiCOW program $\left(H_{0}\right.$ Lenses in COSMOGRAIL's Wellspring), which focuses on improving the detailed 
modeling of the lens galaxy and of the mass along the line of the sight to the background quasar, led to $H_{0}=73.3_{-1.8}^{+1.7} \mathrm{~km} / \mathrm{s} / \mathrm{Mpc}$ (that is $2.4 \%$ precision) in a flat $\Lambda$ CMD Universe by using deep HST imaging, Keck spectroscopy and AO imaging and wide field Subaru imaging [20, 116, 125, 131, 150, 151]. This value is in excellent agreement with the most recent measurements using the distance ladder (though in tension with the CMB measurements from Planck) but still lacks precision.

By performing photometric measurements with the required sensitivity and no interruption, the combination of a high precision astrometric mission and excellent modeling of the lens galaxy, will enable measurement of $H_{0}$ at the percent level and remove any possible degeneracies between $H_{0}$ and other cosmological parameters. This will open up new avenues to test the nature of DM. An alternative technique consists of using trigonometric parallaxes. This is the only (non-statistical and modelindependent) direct measurement method and the foundation of the distance scale. A high precision astrometric mission has the potential to extend the "standard candles" - the more distant pulsating variables: Cepheids, RR Lyrae, Miras and also Stellar Twin stars - well beyond the reach of Gaia.

These distance measurements can be transferred to nearby galaxies allowing us to convert observable quantities, such as angular size and flux, into physical qualities such as energy and luminosity. Importantly, these distances scale linearly with $H_{0}$, which gives the temporal and spatial scale of the Universe. With this improved knowledge, we will then be able to better understand the structure and evolution of both our own and more distant galaxies, and the age of our Universe.

\subsection{Position of the science targets in the sky}

The different targets considered for observations with a high precision astrometry mission have been located in Fig. 15 on a sky map.

\section{Possible space mission}

Several mission profiles have been considered in the last few years focused on differential astrometry, for instance NEAT, micro-NEAT and Theia. Additional new differential astrometry mission configurations adapted with technological innovations will certainly be envisioned to pursue accurate measurements of the extremely small motions required by the science cases in this White Paper.

\subsection{Scientific requirements}

To address the science described in this white paper, a high precision astrometry mission should stare towards:

- dwarf spheroidal galaxies to probe their dark matter inner structure;

- hyper-velocity stars to probe the triaxiality of the halo, the existence of compact minihalo objects and the time delay of quasars;

- the Galactic Disc, to probe DM subhalos and compact minihalo objects; 


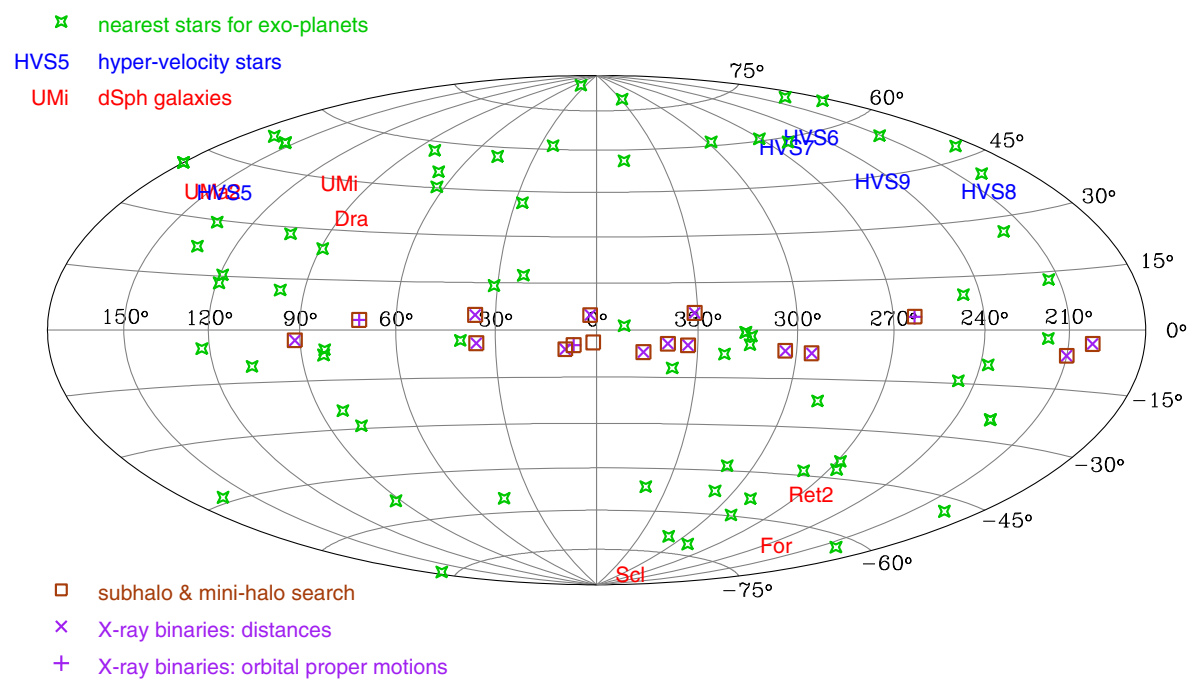

Fig. 15 Sky map of the targets considered for observations with a high precision astrometric mission

- star systems in the vicinity of the Sun, to find the nearest potentially habitable terrestrial planets;

- $\quad$ known X-ray binaries hosting neutron stars or black holes.

For a targeted mission, the objects of interest must be sampled throughout the lifetime of the mission. After re-pointing the telescope and while waiting for stabilization, photometric surveys, e.g. for measurements of $H_{0}$ using lensed quasar time delays could be performed, thus optimizing the mission scientific throughput. Figure 15 shows a sample sky map with potential targets.

As illustrated in Fig. 16, high precision astrometric missions could measure the plane-of-sky velocities of the faintest objects in the local Universe, with errors as small as a few $\mathrm{mm} / \mathrm{s}$ in the case of the hosts of Earth-mass exoplanets in the habitable zone of nearby stars, a few m/s for stars in the Milky Way disc, i.e. for kinematical searches for DM sub-halos, micro-lensing searches for ultra-compact minihaloes, and for the companions of neutron stars and black holes in X-ray binaries, $200 \mathrm{~m} / \mathrm{s}$ for hyper-velocity stars whose line of sight velocities are typically $>500 \mathrm{~km} / \mathrm{s}$, and finally $1 \mathrm{~km} / \mathrm{s}$ for $R=20 \mathrm{mag}$ stars for dwarf spheroidal galaxies.

A mission concept with an expected Theia-like astrometric precision, as shown in Fig. 17, surpasses what will be achieved by other approved space astrometric surveys and ground surveys, thus unlocking science cases that are still unreachable.

Table 1 summarizes the science cases with the most stringent performance requirements. To cover the science questions from this White Paper, any mission concept must be flexible, allowing for observing modes covering a wide flux dynamical range. This requires the concepts to cope with Deep Field Modes, aimed towards objects such as dwarf galaxies, and Bright Star Modes, focused on the study of planetary systems around nearby stars. 


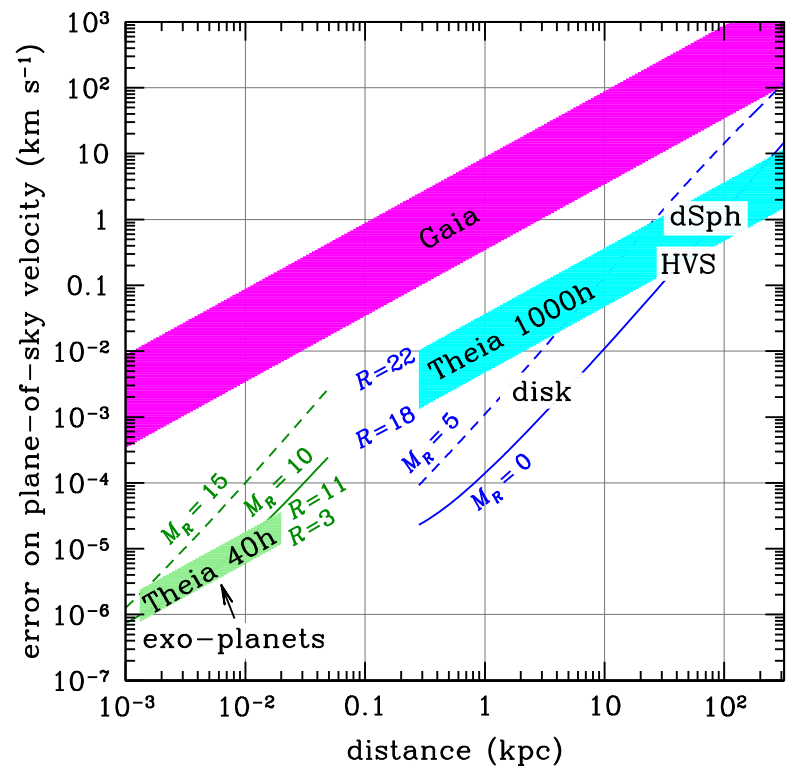

Fig. 16 Expected plane-of-sky velocity errors from a high precision astrometry mission's proper motions as a function of distance from Earth. These errors respectively correspond to 40 and 1000 cumulative hours of exposures for exoplanets (green) and more distant objects (cyan and blue), during a 4 year interval for observations, including the systematic limit from calibration on Gaia reference stars. The expected precision for specific objects are highlighted. The accuracy for the 5-year Gaia mission is shown in magenta
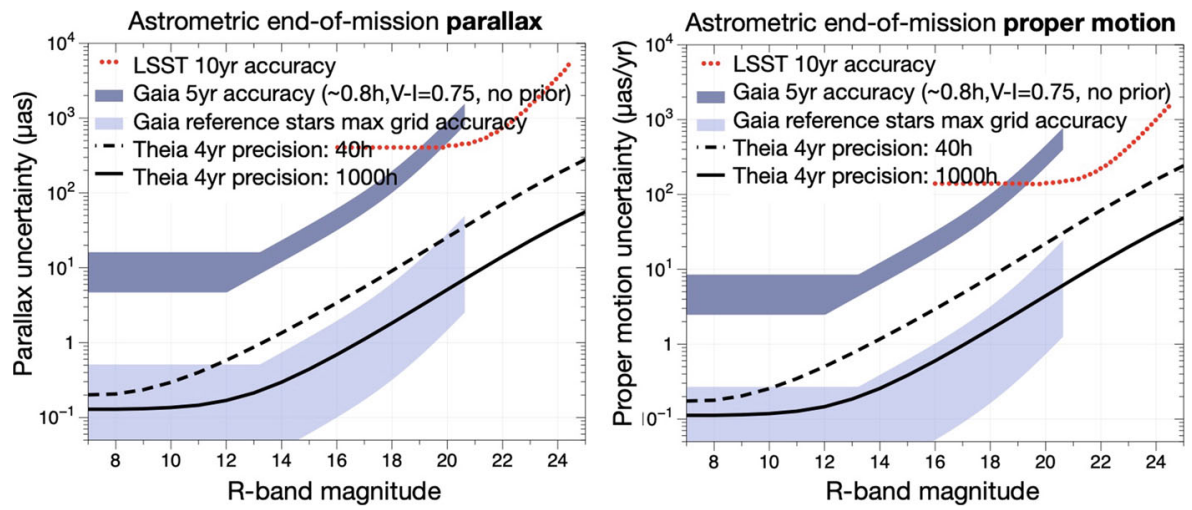

Fig. 17 Estimated RMS precision on a high precision astrometry mission relative parallax (left, for ecliptic latitude $0^{\circ}$ ) and proper motion (right) in the $R$-band. Also shown for comparison are the estimated accuracies for 10 years LSST [83] as well as the 5-year nominal Gaia mission [34] (vertical spread caused by position on the sky, star color, and bright-star observing conditions). Small-scale spatial correlations $\left(<1^{\circ}\right)$ between Gaia reference sources will limit the maximum reachable absolute parallax and proper motion calibration for a high precision astrometry mission [61, 62], indicated by the light blue band for a range of assumed spatial correlations (expected to be much below $r=0.5 \%$; [60]) as a function of reference star magnitude. Bright stars $(V<13)$ and low star-density regions will have the highest correlations 
Table 1 Summary of science cases with most stringent performance requirements set in each case

\begin{tabular}{|c|c|c|c|c|c|}
\hline Program & $\begin{array}{l}\text { Used } \\
\text { time (h) }\end{array}$ & $\begin{array}{l}\text { Mission } \\
\text { fraction }\end{array}$ & $\begin{array}{l}\text { Number of } \\
\text { objects per field }\end{array}$ & $\begin{array}{l}\text { Benchmark target } \\
R \text { mag (and range) }\end{array}$ & $\begin{array}{l}\text { EoM precision } \\
\text { (at ref. mag.) }\end{array}$ \\
\hline Dark Matter & 17000 & 0.69 & $10^{2}-10^{5}$ & $20(14-22)$ & $10 \mu$ as \\
\hline \multicolumn{6}{|l|}{ \& compact objects } \\
\hline $\begin{array}{l}\text { Nearby Earth-like } \\
\text { planets \& follow-up }\end{array}$ & 3500 & 0.14 & $<20$ & $5(1-18)$ & $0.15 \mu$ as \\
\hline Open observatory & 4000 & 0.17 & $10-10^{5}$ & $6(1-22)$ & $1.0 \mu \mathrm{as}$ \\
\hline Overall requirements & 24500 & 1.00 & $10^{1}-10^{5}$ & $6(1-22)$ & $0.15-10 \mu$ as \\
\hline
\end{tabular}

Figures are based on a 4 year mission, thermal stabilisation (+slew time) is assumed to take $30 \%$ of the mission time

\subsection{Example of a medium-size mission}

The Payload Module (PLM) of a high precision astrometric mission must be simple. It is essentially composed of four subsystems: telescope, camera, focal plane array metrology and telescope metrology. In the case of the Theia/M5 concept, they were designed applying heritage from space missions and concepts like Gaia, HST/FGS, SIM, NEAT (proposed for the ESA M3 opportunity), Theia (proposed for the ESA M4 opportunity), and Euclid.

However, achieving microarcsecond differential astrometric precision requires the control of all effects that can impact the determination of the relative positions of the point spread function. The typical apparent size of an unresolved star corresponds to 0.2 arcseconds for a $0.8 \mathrm{~m}$ telescope operating in visible wavelengths. The challenge is therefore to control systematic effects to the level of 1 part per 200000 . The precision of relative position determination in the Focal Plane Array (FPA) depends on i) the photon noise, which can be either dominated by the target or by the reference stars; ii) the geometrical stability of the instrument, iii) the stability of the optical aberrations, and iv) the variation of the detector quantum efficiency between pixels. The control of these effects impairs other missions that otherwise could perform microarcsecond differential astrometry measurements, like HST, Kepler, the Roman Space Observatory (previously known as WFIRST), or Euclid, posing fundamental limits to their astrometric accuracy. All these effects must be taken into account in any high precision differential astrometry mission concept.

To address the challenges and fulfil the requirements from Section 2.1, two different possible concepts can be investigated. A NEAT-like mission consisting of a formation flight configuration [87] or an Euclid-like mission, ${ }^{5}$ but with a single focal plane and additional metrology subsystems. Both concepts are based on adopting a long focal length, diffraction-limited telescope, and additional metrological control of the focal plane array. The proposed Theia/M5 mission concept was the result of a trade-off analysis between both concepts.

\footnotetext{
${ }^{5}$ Euclid red book: http://sci.esa.int/euclid/48983-euclid -definition-study-report-esa-sre-2011-12.
} 


\subsubsection{Telescope concept}

The Theia PLM concept consists of a single Three Mirror Anastigmatic (TMA) telescope with a single focal plane (see Fig. 18) covering a $0.5^{\circ}$ field-of-view with a mosaic of detectors. To monitor the mosaic geometry and its quantum efficiency, the PLM includes a focal plane metrology subsystem, while to monitor the telescope geometry, a dedicated telescope metrology subsystem is used.

To reach sub-microarcsecond differential astrometry a diffraction-limited telescope, with all aberrations controlled, is necessary. A trade-off analysis was performed between different optical designs, which resulted in two optical concepts that could fulfil all requirements. Both are based on a Korsch TMA telescope; one is an on-axis solution while the second is an off-axis telescope. In both cases only three of the mirrors are powered mirrors. While the on-axis solution adopts a single folding mirror, the off-axis solution adopts two folding mirrors. The on-axis design was the Theia/M5 baseline (Fig. 19). More recently, however, studies from NASA/JPL show that a customized and corrected Ritchey-Chrétien can reach $5 \mu$ as over a $0.5^{\circ} \mathrm{FoV}$, which even if not capable of addressing habitable exoplanet science cases, would provide a valuable instrument for Dark Matter studies.

To achieve the precision by centroiding as many stars as possible, a mosaic of detectors (in principle CCD or CMOS) must be assembled on the focal plane (Fig. 20). The detectors must feature small pixels $(\sim 10 \mu \mathrm{m})$ and well controlled systematic errors along the lifetime of the mission. Detailed in-orbit calibration of the focal plane and detector geometry and response must be monitored, and in the Theia concept this is addressed via a dedicated laser metrology (see Section 4.3).

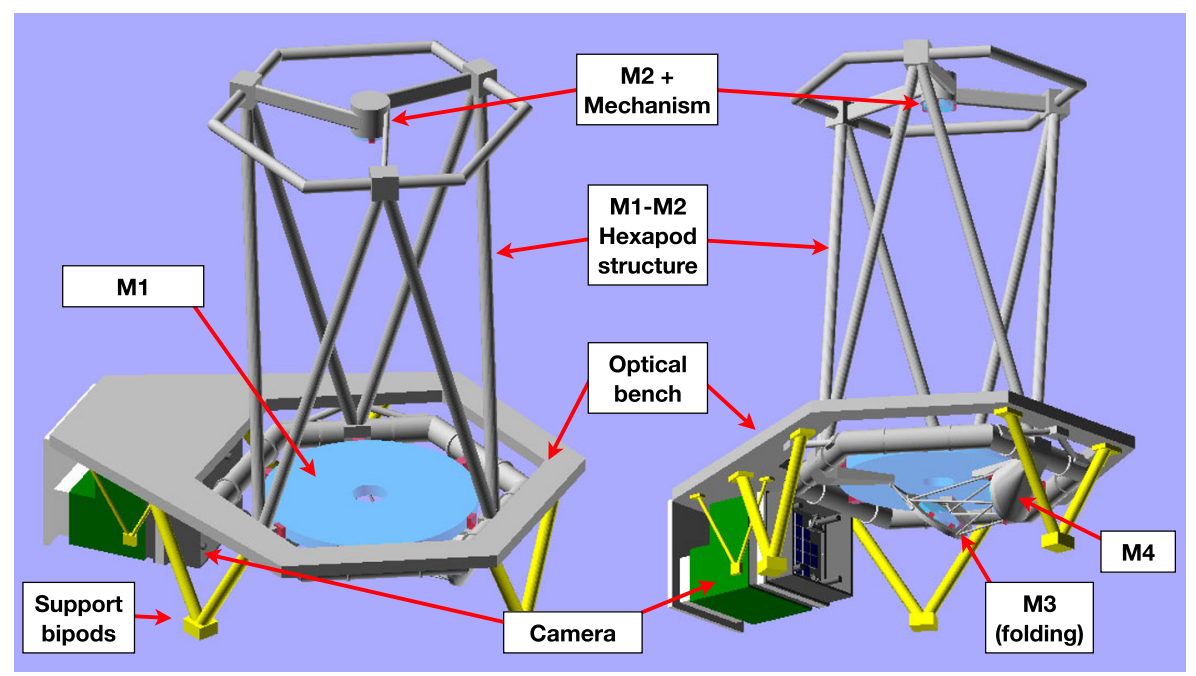

Fig. 18 Overall layout of the Theia Payload Module concept. Volume is estimated at $1.6 \times 1.9 \times 2.2 \mathrm{~m}^{3}$. 

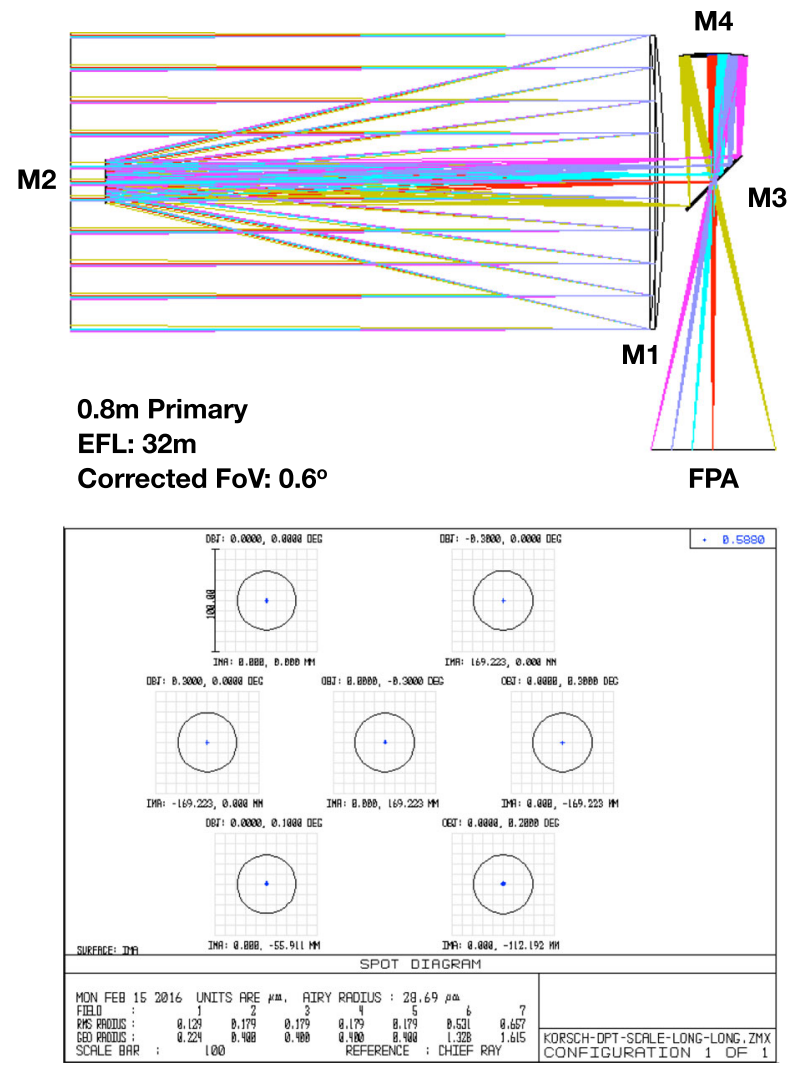

Fig. 19 On-axis Korsch TMA option. Ray-tracing and spot diagrams for the entire FoV. This design was adopted as the baseline for the Theia/M5 proposal. EFL is Effective Focal Length
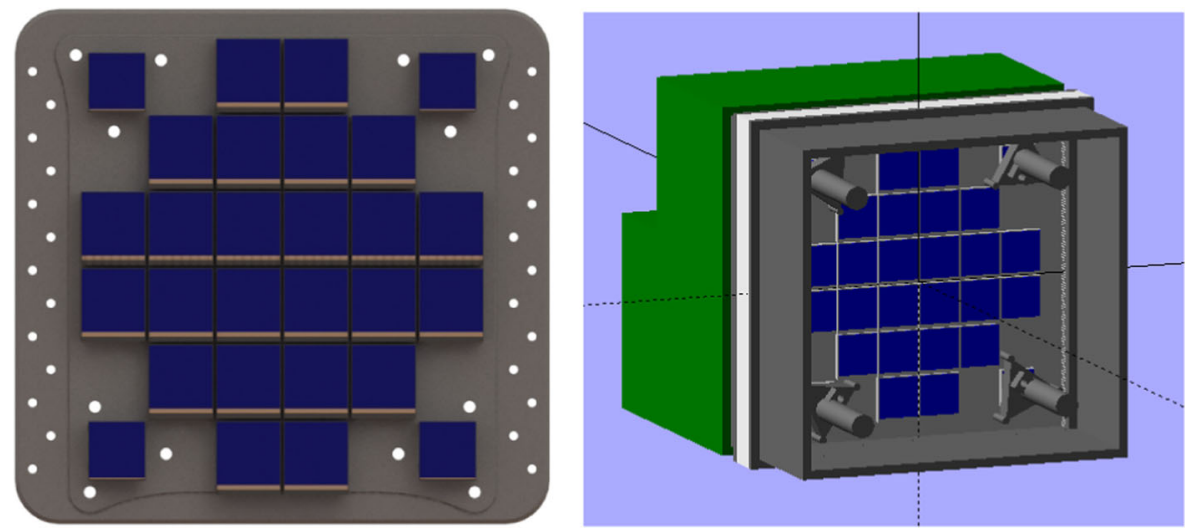

Fig. 20 Concept for the Theia/M5 Camera. Left: concept for the FPA detector plate. Right: overall view of the camera concept 
Table 2 Theia's mission main characteristics

\begin{tabular}{ll}
\hline Launch date & No constraints, allowing launch date in 2029 \\
Orbit & Large Lissajous in L2 \\
Lifetime & $\bullet 4$ years of nominal science operations \\
& - Technical operations: 6 months orbit transfer plus instrument \\
& commissioning and 1 month decommissioning \\
Concept & Single spacecraft, single telescope in the PLM, single camera \\
& in the focal plane, metrological monitoring of PLM \\
Communication architecture & $75 \mathrm{Mbps}, 4 \mathrm{~h} /$ day \\
\hline
\end{tabular}

\subsubsection{Mission configuration and profile}

The time baseline (Table 2) to properly investigate the science topics of this White Paper would be at least 4 years, including time devoted to orbit maintenance. A total of approximately 6 months has been estimated for the orbit transfer including the spacecraft and instrument commissioning. This estimate is made from the total of $\sim 35000 \mathrm{~h}$ corresponding to the total time for the scientific program (Table 1) and considering that about 15 min per slew will be dedicated to reconfiguration and station-keeping, while thermal stabilization time is in addition to the slew time.

Some instrument key features of the Theia concept are presented in Fig. 21. The concept is inspired by the Euclid service module with a downscaled size to minimize mass and improve mechanical properties. Similar to the Euclid and Herschel satellites, Theia's Korsch telescope is accommodated on top of the service module in a vertical position leading to a spacecraft height of about $5 \mathrm{~m}$. This concept optimizes the payload size.

\section{Worldwide context of ground-based and space science}

Observations carried out with a mission dedicated to high precision astrometry will add significant value and will benefit from a number of other ground-based and space missions operating in the 2030s and beyond, including ESA's Athena, PLATO, Euclid and Gaia missions, ESO's MICADO and Gravity instruments, CTA, SKA, the NASA/ESA/CSA JWST and the Rubin Observatory (previously known as LSST). For example:

- JWST: Estimates suggest that JWST will be able to detect Lyman Break galaxies with absolute magnitudes as faint as $M_{\mathrm{UV}} \sim-15$ at $z \sim 7$ [33], corresponding to halo masses of about $10^{9.5} M_{\odot}$. The combination of a high precision astrometry mission and JWST's observations will enable unambiguous tests of DM.

- PLATO: this mission will look at planetary transits and star oscillations in two fields (each covering $2250 \mathrm{deg}^{2}$ ), for 2-3 years each, in host stars brighter than 16 mag. PLATO high cadence continuous monitoring of its target stars will provide information on the internal structure of the stars, allowing determination of their stellar ages and masses. A high precision astrometry mission will benefit from 


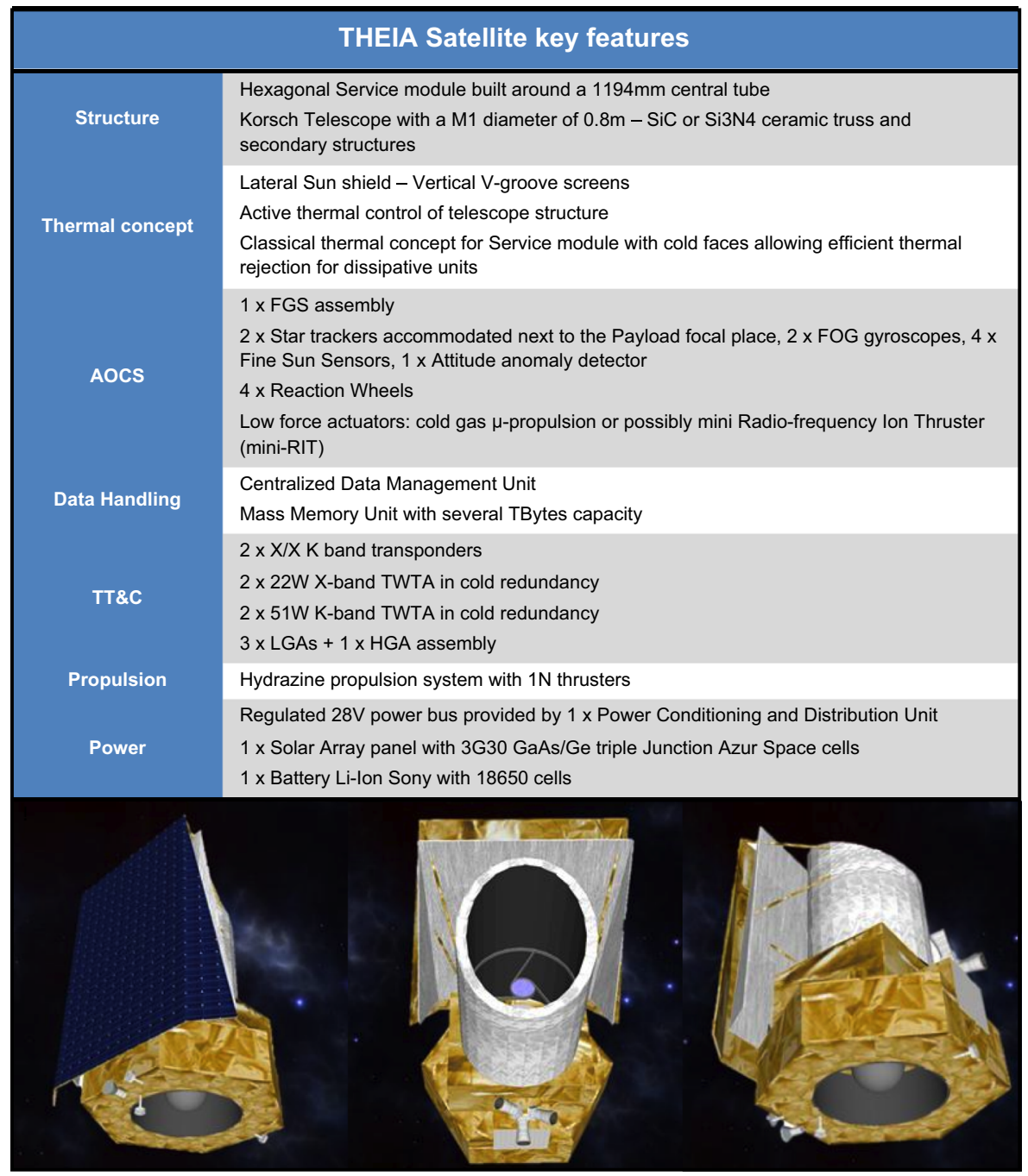

Fig. 21 Proposed Theia satellite concept (Thales Alenia Space). FGS: Fine Guidance Sensor; FOG: Fiber Optics Gyroscope; AOCS: Attitude and Orbit Control System,TT\&C: Telemetry, Tracking \& Control; TWTA: Travelling Wave Tube amplifier Assembly; LGA: Low Gain Antenna; HGA: High Gain Antenna

PLATO characterization of many of the astrometry mission's core star samples. For close PLATO stars where transits were observed this astrometry mission can measure additional inclined planets.

- SKA: SKA aims to use radio signals to look for building blocks of life (e.g. amino acids) in Earth-sized planets $[58,155]$. A high precision astrometry will identify target planets from their astrometric "wobble" that can be followed-up spectroscopically with the SKA. Furthermore, SKA aims to use its immensely fast sky coverage to detect transients [40], such as supernovae and gamma-ray 
bursts. With its precise astrometry, Theia will help study the specific locations of such events in stellar clusters.

- CTA: The Cherenkov Telescope Array (CTA) in the Northern and Southern Hemispheres will carry out measurements of the gamma-ray flux with almost complete sky coverage and unprecedented energy and angular resolution, in the $\sim 0.02-100 \mathrm{TeV}$ energy range [7]. The sub-microarc-second performance of a high precision astrometry mission allow us investigating the so-called J-factor which corresponds to the brightness of the gamma-ray flux in dwarf galaxies and thus determines the prime candidates for CTA's observations. CTA also aims at observing star forming systems over six orders of magnitude in formation rate, to measure the fraction of interacting cosmic rays as a function of the starformation rate. By combining high precision astrometry and CTA measurements, we will better understand the relative importance of cosmic rays and DM in places where star-formation is important. Furthermore, a small number of black hole and neutron star binary systems in our Galaxy are known to emit gamma rays. The mechanism by which the particle acceleration is achieved is not well understood. The sub-microarcsecond performance of a high precision astrometry mission will allow us to probe the velocity structure of the nearby gamma-ray bright radio galaxies of NGC 1275, IC 310, M 87, and Cen A, which combined with CTA's observations will enable important astrophysics breakthroughs.

\section{Technology challenges for high precision astrometry}

\subsection{Spacecraft technology and cost}

There have been several propositions for a space mission dedicated to high precision astrometry: a 6 meter baseline visible interferometer on a single satellite like SIM or SIM-Lite [47]; a single mirror off-axis parabola $1 \mathrm{~m}$-diameter telescope based on two spacecraft, one carrying the telescope mirror and the other the focal plane like the NEAT telescope [87]; or a single-mirror telescope like Theia [17, 86]. The variety of the concepts shows that there are areas of progress on spacecraft technologies, especially concerning formation flying, actively-controlled large structure interferometers.

One interesting potential solution to be considered is the nanosat technology and the cost reduction that is linked to it. There is a huge cost difference between cubesats $(<10 \mathrm{M} €)$ and an ESA M-class mission $(400-500 \mathrm{M} €)$ or NASA MIDEX/Discovery mission (300-500 M\$). The cubesat technology has matured and many hundreds are launched every year. That technology has now crept into microsats that are up to $200 \mathrm{~kg}$ and spacecraft bus of this category are now $<5 \mathrm{M} €$, while only a few years ago they were $\sim 40 \mathrm{M} €$. Because of their low cost and the high number of flying satellites, this technology has now demonstrated 5 year typical lifetime, comparable to a more expensive traditional spacecraft. In any case, all the price scales will change between now and the epoch when Voyage 2050 will be implemented and that includes flying heavier payloads because of the decrease of launch costs [67]. 


\subsection{Detection}

Presently, two detector technologies are used: CCD or CMOS. CMOS detectors present a high quantum efficiency over a large visible spectral band that can also reach infrared wavelengths depending on the sensitive layer. CMOS detectors also have programmable readout modes, faster readout, lower power, better radiation hardness, and the ability to put specialized processing within each pixel. On the other hand there are many known detector systematics, even for advanced detectors like the Teledyne H4RG10. The main challenging effects are the following: fluence-dependent PSF, correlated read noise, inhomogeneity in electric field lines and persistence effects (e.g. [124]). All mission proposals so far were based on CCD technology, but detector evolution will certainly take place on the context of any mission concept to answer the challenges being posed by the Voyage 2050 White Papers.

If a Theia-like mission is selected for the 2040's, detector technology might be different from anything we have in place nowadays. The main requirements are small pixels, low read-out noise (RON) on large format focal plane and mastering intrapixels effects in order to reach the highest precision astrometry. It should be noticed that the development of European detector technology for low-RON and large-format IR and visible detector matrices, like the Alfa detector that ESA is undertaking with Lynred, is of high interest for our science cases.

\subsection{Metrology}

Traditionally systematic errors have been the major challenge for $\mu$ as-level astrometry from space. Astrometric accuracy has a lot in common with photometric accuracy, and the technology development that proceeded the Kepler mission demonstrated $\sim 10^{-5}$ relative photometry. Similar advances have been made in detector calibration for astrometry [31]. Photons from stars carry the astrometric information at exquisite precision, systematic errors are imparted when those photons strike the telescope optics and also when they are detected by the focal plane array. The calibration of optical field distortion using reference stars is a technique that is perhaps a century old and used on ground and space-based telescopes.

Metrology laser-feed optical fibers placed at the back of the nearest mirror to the detectors can be used to monitor distortions of the focal plane array, and to allow the associated systematic errors to be corrected [31]. Such detector calibration at $10^{-6}$ pixel levels should be continued. In addition to measuring the FPA physical shape, the rest of the telescope needs monitoring to control time-variable aberrations at sub $\mu$ as level. Even at very stable space environments such as L2, the telescope geometry is expected to vary for different reasons: structural lattice reorganization (such as the micro-clanks observed in ESA's Gaia mission), outgassing and most importantly, thermo-elastic effects due to the necessary variation of the Solar Aspect Angle during the mission for pointings to the different science targets.

In the case of Theia, the telescope metrology subsystem to monitor perturbations to the telescope geometry is based on a concept of a series of simple and independent linear displacement interferometers installed between the telescope mirrors and organized in a virtual hexapod configuration. Existing space-based interferometers 
from TNO, as the Gaia Basic Angle Monitor (BAM) are already capable of reaching more precise measurements than those required by Theia/M5 - BAM can perform $\sim 1.5$ pm optical path difference measurements [44]. A Thales telemeter developed for CNES can reach $\sim 100 \mathrm{pm}$, and the Thales interferometer produced for the MTG (Meteosat Third Generation) satellite can reach $1 \mathrm{~nm}$ per measurement [119] - higher precisions can be reached by averaging over many measurements.

For telescopes that do not have high stability levels, there are some alternatives. One is the diffractive pupil concept that puts a precision array of dots on the primary, which produces a regular pattern of dots in the focal plane. One way to use the diffractive pupil is to look at a very bright star $(0 \mathrm{mag})$ and record the diffraction pattern interspersed with observations of a much dimmer target star ( $\sim 7 \mathrm{mag})$. The diffractive pupil can also be used during science observations, but when the target star is $\sim 7$ mag photon noise of the diffracted light can be significantly higher than the photon noise of the reference stars $(\sim 11-14 \mathrm{mag})$.

\section{Conclusion}

To solve fundamental questions like

- "What is the nature of dark matter?"

- "Are there habitable exo-Earths nearby?"

- "What is the equation of state of matter in extreme environments?"

- "Can we put direct constraints on cosmological models and dark energy parameters?"

many branches of astronomy need to monitor the motion of faint objects with significantly higher precision than what is accessible today. Through ultra-precise microarcsecond relative astrometry, a high precision astrometry space mission will address the large number of important open questions that have been detailed in this White Paper.

The scientific requirements points toward a space mission that is relatively simple: a single telescope, with metrology subsystems and a camera. Such a mission can fit as an M-class mission, or even at a smaller mission class depending on the final accuracy which is desired.

Some technological challenges must be tackled and advanced: the spacecraft, the focal plane detector and the metrology. We believe that these challenges can be mastered well before 2050 and that they will open the compelling scientific window of the faint objects in motion.

Acknowledgements The authors would like to thank the researchers and engineers who are not coauthors of this paper but who have taken part and have brought their contribution to the proposed missions to ESA successive calls: NEAT (M3), micro-NEAT (S1), and Theia (M4 and M5). An extensive list of supporters for the science objectives is given in [17]. We thank also Arianna Gallo for her contribution in our investigation of the shape of the Milky Way dark matter halo and Krzysztof A. Rybicki who generated the plots from Fig. 14.

We are grateful to the anonymous referee who helped to improve the quality of the paper with his/her remarks. 
Concerning the funding of our work, we would like to acknowledge the support of many agencies or programs. R.B. acknowledges support from NASA's Virtual Planetary Laboratory lead team under cooperative agreements NNA13AA93A. A.C.M.C. acknowledges support from CFisUC strategic project (UID/FIS/04564/2019). F.C. acknowledges support by the Swiss National Science Foundation (SNSF) and by the European Research Council (ERC) under the European Union's Horizon 2020 research and innovation program (COSMICLENS: grant agreement No. 787886). M.F. received support from Polish National Science Centre (NCN) under Grant No. 2017/26/D/ST9/00591. M.F. gratefully acknowledge the support of the Swedish National Space Agency (DNR 65/19, 174/18). D.H. thanks the Swedish National Space Agency (SNSA/Rymdstyrelsen) for their support. A.M. thanks the Portugese Fundação para a Ciência e a Tecnologia (FCT) through the Strategic Programme UID/FIS/00099/2019 for CENTRA. P.S. acknowledges support from the Australian Research Council under grant FT190100814. L.W. acknowledges support from the Polish NCN grants: Harmonia No. 2018/06M/ST9/00311 and Daina No. 2017/27/L/ST9/03221. The OATo team acknowledges partial funding by the Italian Space Agency (ASI) under contracts 2014-025-R.1.2015 and 2018-24-HH.0, and by a grant from the Italian Ministry of Foreign Affairs and International Cooperation (ASTRA). A.C. and F.M. acknowledge support by the LabEx FOCUS ANR-11-LABX-0013. The work of C.J., X.L. and J.P. was supported by the Spanish Ministry of Science, Innovation and University (MICIU/FEDER, UE) through grants RTI2018-095076-B-C21, ESP2016-80079-C2-1-R, and the Institute of Cosmos Sciences University of Barcelona (ICCUB, Unidad de Excelencia 'María de Maeztu') through grants MDM-2014-0369 and CEX2019-000918-M. A.K.-M., A.A., V.C., P.G., P.G., A.M.A., A.M., M.S. were supported by Fundação para a Ciência e a Tecnologia, with grants reference UIDB/00099/ 2020 and SFRH/BSAB/142940/2018 (P.G. only). A.D. and L.O. also acknowledge partial support from the Italian Ministry of Education, University and Research (MIUR) under the Departments of Excellence grant L.232/2016, and from the INFN grant InDark. G.J.W. gratefully acknowledges support of an Emeritus Fellowship from The Leverhulme Trust. EV is supported by Spanish grant PGC2018-101950-B-100.

This research has made use of NASA's Astrophysics Data System Bibliographic Services.

Funding Open access funding provided by Istituto Nazionale di Astrofisica within the CRUI-CARE Agreement.

Open Access This article is licensed under a Creative Commons Attribution 4.0 International License, which permits use, sharing, adaptation, distribution and reproduction in any medium or format, as long as you give appropriate credit to the original author(s) and the source, provide a link to the Creative Commons licence, and indicate if changes were made. The images or other third party material in this article are included in the article's Creative Commons licence, unless indicated otherwise in a credit line to the material. If material is not included in the article's Creative Commons licence and your intended use is not permitted by statutory regulation or exceeds the permitted use, you will need to obtain permission directly from the copyright holder. To view a copy of this licence, visit http://creativecommons.org/licenses/by/4.0/.

\section{References}

1. Abbott, B.P., Abbott, R., Abbott, T.D., et al.: Observation of Gravitational Waves from a Binary Black Hole Merger. Phys. Rev. Lett. 116, 061102 (2016)

2. Abbott, B.P., Abbott, R., Abbott, T.D., et al.: The Rate of Binary Black Hole Mergers Inferred from Advanced LIGO Observations Surrounding GW150914. ApJ 833, L1 (2016)

3. Abbott, B.P., Abbott, R., Abbott, T.D., et al.: GWTC-1: A Gravitational-Wave Transient Catalog of Compact Binary Mergers Observed by LIGO and Virgo during the First and Second Observing Runs. Physical Review X 9, 031040 (2019)

4. Abbott, B.P., Abbott, R., Abbott, T.D., et al.: Search for Post-Merger Gravitational Waves from the Remnant of the Binary Neutron Star Merger GW170817. ApJ 851, L16 (2017)

5. Abbott, B.P., Abbott, R., Adhikari, R., et al.: LIGO: the Laser Interferometer Gravitational-Wave Observatory. Reports on Progress in Physics 72, 076901 (2009)

6. Acernese, F., Agathos, M., Agatsuma, K., et al.: Advanced Virgo: a Second-Generation InterferoMetric Gravitational Wave Detector. Classical and Quantum Gravity 32, 024001 (2015) 
7. Actis, M., Agnetta, G., Aharonian, F., et al.: Design Concepts for the Cherenkov Telescope Array CTA: an Advanced Facility for Ground-Based High-Energy Gamma-Ray Astronomy. Exp. Astron. 32, 193 (2011)

8. Alam, S., Ho, S., Silvestri, A.: Testing Deviations from $\Lambda$ CDM with Growth Rate Measurements from Six Large-Scale Structure Surveys at $\mathrm{z}=0.06-1$. MNRAS 456, 3743 (2016)

9. Anglada-Escudé, G., Amado, P.J., Barnes, J., et al.: A Terrestrial Planet Candidate in a Temperate Orbit around Proxima Centauri. Nature 536, 437 (2016)

10. Antoniadis, J., Freire, P.C.C., Wex, N., et al.: A Massive Pulsar in a Compact Relativistic Binary. Science 340, 448 (2013)

11. Armstrong, D.J., Osborn, H.P., Brown, D.J.A., et al.: On the Abundance of Circumbinary Planets. MNRAS 444, 1873 (2014)

12. Arzoumanian, Z., Brazier, A., Burke-Spolaor, S., et al.: The NANOGrav 11-year Data Set: Highprecision Timing of 45 Millisecond Pulsars. ApJS 235, 37 (2018)

13. Aslanyan, G., Price, L.C., Adams, J., et al.: Ultracompact Minihalos as Probes of Inflationary Cosmology. Phys. Rev. Lett. 117, 141102 (2016)

14. Banik, I., Kroupa, P.: Directly Testing Gravity with Proxima Centauri. MNRAS 487, 1653 (2019)

15. Binney, J., Mamon, G.A.: M/L and Velocity Anisotropy from Observations of Spherical Galaxies, or Must M87 have a Massive Black Hole. MNRAS 200, 361 (1982)

16. Bird, S., Peiris, H.V., Viel, M., Verde, L.: Minimally Parametric Power Spectrum Reconstruction from the Lyman $\alpha$ Forest. MNRAS 413, 1717 (2011)

17. Boehm, C., Krone-Martins, A., Amorim, A., et al.: Theia: Faint Objects in Motion or the new Astrometry Frontier. arXiv:1707.01348 (2017)

18. Boehm, C., Schewtschenko, J.A., Wilkinson, R.J., Baugh, C.M., Pascoli, S.: Using the Milky Way Satellites to Study Interactions Between cold Dark Matter and Radiation. MNRAS 445, L31 (2014)

19. Bonaca, A., Conroy, C., Price-Whelan, A.M., Hogg, D.W.: Multiple Components of the Jhelum Stellar Stream. ApJ 881, L37 (2019)

20. Bonvin, V., Courbin, F., Suyu, S.H., et al.: MNRAS (2016)

21. Brasser, R., Ida, S., Kokubo, E.: A Dynamical Study on the Habitability of Terrestrial Exoplanets I. Tidally Evolved Planet-Satellite Pairs. MNRAS 428, 1673 (2013)

22. Bringmann, T., Scott, P., Akrami, Y.: Improved Constraints on the Primordial Power Spectrum at Small Scales from Ultracompact Minihalos. Phys. Rev. D 85, 125027 (2012)

23. Brown, W.R.: Hypervelocity stars. ARA\&A 53, 15 (2015)

24. Brown, W.R., Anderson, J., Gnedin, O.Y., et al.: Proper Motions and Trajectories for 16 Extreme Runaway and Hypervelocity Stars. ApJ 804, 49 (2015)

25. Brown, W.R., Geller, M.J., Kenyon, S.J., Kurtz, M.J.: Discovery of an Unbound Hypervelocity Star in the Milky Way Halo. ApJ 622, L33 (2005)

26. Brown, W.R., Lattanzi, M.G., Kenyon, S.J., Geller, M.J.: Gaia and the Galactic Center Origin of Hypervelocity Stars. ApJ 866, 39 (2018)

27. Bryan, S.E., Mao, S., Kay, S.T., et al.: Influence of Baryons on the Orbital Structure of Dark Matter Haloes. MNRAS 422, 1863 (2012)

28. Carlberg, R.G.: The Density Structure of Simulated Stellar Streams. ApJ 889, 107 (2020)

29. Chluba, J., Erickcek, A.L., Ben-Dayan, I.: Probing the Inflaton: Small-Scale Power Spectrum Constraints from Measurements of the Cosmic Microwave Background Energy Spectrum. ApJ 758, 76 (2012)

30. Cromartie, H.T., Fonseca, E., Ransom, S.M., et al.: Relativistic Shapiro Delay Measurements of an Extremely Massive Millisecond Pulsar. Nature Astronomy 4, 72 (2020)

31. Crouzier, A., Malbet, F., Hénault, F., et al.: The Latest Results from DICE (Detector Interferometric Calibration Experiment). Proc. SPIE 9904, 99045G (2016)

32. Damasso, M., Sozzetti, A., Lovis, C., et al.: A Precise Architecture Characterization of the $\pi$ Mensae Planetary System. A\&A 642, A31 (2020)

33. Dayal, P., Dunlop, J.S., Maio, U., Ciardi, B.: Simulating the Assembly of Galaxies at Redshifts $\mathrm{z}=$ 6-12. MNRAS 434, 1486 (2013)

34. de Bruijne, J.H.J., Rygl, K.L.J., Antoja, T.: In: EAS Publications Series, vol. 67, pp. 23-29 (2014)

35. Demorest, P.B., Pennucci, T., Ransom, S.M., Roberts, M.S.E., Hessels, J.W.T.: A Two-Solar-Mass Neutron Star Measured using Shapiro Delay. Nature 467, 1081 (2010)

36. Dubinski, J.: The Effect of Dissipation on the Shapes of Dark Halos. ApJ 431, 617 (1994)

37. Edelmann, H., Napiwotzki, R., Heber, U., Christlieb, N., Reimers, D.: HE 0437-5439: an Unbound Hypervelocity Main-Sequence B-Type Star. ApJ 634, L181 (2005) 
38. Erkal, D., Li, T.S., Koposov, S.E., et al.: Modelling the Tucana III Stream - a Close Passage with the LMC. MNRAS 481, 3148 (2018)

39. Feldmann, R., Spolyar, D.: Detecting Dark Matter Substructures Around the Milky Way with Gaia. MNRAS 446, 1000 (2015)

40. Fender, R., Stewart, A., Macquart, J.P., et al.: The Transient Universe with the Square Kilometre Array. In: Advancing Astrophysics with the Square Kilometre Array (AASKA14), 51 (2015)

41. Fortin, M. et al.: Neutron Star Radii and Crusts: Uncertainties and Unified Equations of State. Phys. Rev. C 94, 035804 (2016)

42. Gaia Collaboration, Prusti, T., de Bruijne, J.H.J., et al.: The Gaia Mission. A\&A 595, A1 (2016)

43. Gallo, A., Chakrabarty, S.S., Ostorero, L., Ebagezio, S., Diaferio, A.: ApJ, in preparation (2021)

44. Gielesen, W., de Bruijn, D., van den Dool, T., et al.: Gaia Basic Angle Monitoring System. Proc. SPIE 8863, 88630G (2013)

45. Gillon, M., Jehin, E., Lederer, S.M., et al.: Temperate Earth-Sized Planets Transiting a Nearby Ultracool Dwarf Star. Nature 533, 221 (2016)

46. Gnedin, O.Y., Gould, A., Miralda-Escudé, J., Zentner, A.R.: Probing the Shape of the Galactic Halo with Hypervelocity Stars. ApJ 634, 344 (2005)

47. Goullioud, R., Catanzarite, J.H., Dekens, F.G., Shao, M., Marr, J.C.I.: Overview of the SIM PlanetQuest Light Mission Concept. In: Proc. SPIE, vol. 7013, p. 70134T (2008)

48. Haghighipour, N.: On the Dynamical Stability of $\gamma$ Cephei, an S-Type Binary Planetary System. In: Holt, S.S., Deming, D. (eds.) AIP Conf. Ser., vol. 713, The Search for Other Worlds, pp. 269-272 (2004)

49. Haensel, P., Bejger, M., Fortin, M., Zdunik, L.: Rotating Neutron Stars with Exotic Cores: Masses, Radii, Stability. European Phys. J. A 52, 59 (2016)

50. Hattori, K., Valluri, M., Castro, N.: Constraining Solar Position and Velocity with a nearby Hypervelocity Star. ApJ 869, 33 (2018)

51. He, M.Y., Triaud, A.H.M.J., Gillon, M.: First Limits on the Occurrence Rate of Short-Period Planets Orbiting Brown Dwarfs. MNRAS 464, 2687 (2017)

52. Heinke, C.O., Cohn, H.N., Lugger, P.M., et al.: Improved Mass and Radius Constraints for Quiescent Neutron Stars in $\omega$ Cen and NGC 6397. MNRAS 444, 443 (2014)

53. Herzog-Arbeitman, J., Lisanti, M., Madau, P., Necib, L.: Empirical Determination of Dark Matter Velocities Using Metal-Poor Stars. Phys. Rev. Lett. 120, 041102 (2018)

54. Herzog-Arbeitman, J., Lisanti, M., Necib, L.: The Metal-Poor Stellar halo in RAVE-TGAS and its Implications for the Velocity Distribution of Dark Matter. J. Cosmol. Astropart. Phys. 2018, 052 (2018)

55. Hills, J.G.: Hyper-Velocity and Tidal Stars from Binaries Disrupted by a Massive Galactic Black Hole. Nature 331, 687 (1988)

56. Hirsch, H.A., Heber, U., O'Toole, S.J., Bresolin, F.: <ASTROBJ $>$ US $708</ A S T R O B J>~-~ a n$ Unbound Hyper-Velocity Subluminous O Star. A\&A 444, L61 (2005)

57. Hlozek, R., Dunkley, J., Addison, G., et al.: The Atacama Cosmology Telescope: A Measurement of the Primordial Power Spectrum. ApJ 749, 90 (2012)

58. Hoare, M., Perez, L., Bourke, T.L., et al.: SKA and the Cradle of Life. In: Advancing Astrophysics with the Square Kilometre Array (AASKA14), 115 (2015)

59. Høg, E., Fabricius, C., Makarov, V.V., et al.: The Tycho-2 Catalogue of the 2.5 Million Brightest Stars. A\&A 355, L27 (2000)

60. Holl, B., Hobbs, D., Lindegren, L.: Spatial Correlations in the Gaia Astrometric Solution. In: IAU symposium \#261, vol. 261, p. 1703. American Astronomical Society (2009)

61. Holl, B., Lindegren, L.: Error Characterization of the Gaia astrometric solution. I. Mathematical basis of the Covariance Expansion Model. A\&A 543, A14 (2012)

62. Holl, B., Lindegren, L., Hobbs, D.: Error Characterization of the Gaia Astrometric Solution. II. Validating the Covariance Expansion Model. A\&A 543, A15 (2012)

63. Howard, A.W.: Observed Properties of Extrasolar Planets. Science 340, 572 (2013)

64. Howell, S.B., Sobeck, C., Haas, M., et al.: The K2 Mission: Characterization and Early Results. PASP 126, 398 (2014)

65. Irrgang, A., Geier, S., Heber, U., Kupfer, T., Fürst, F.: PG 1610+062: a Runaway B Star Challenging Classical Ejection Mechanisms. A\&A 628, L5 (2019)

66. Irrgang, A., Kreuzer, S., Heber, U.: Hypervelocity Stars in the Gaia era. Runaway B Stars beyond the Velocity Limit of Classical Ejection Mechanisms. A\&A 620, A48 (2018) 
67. Jones, H.W.: The Recent Large Reduction in Space Launch Cost. In: 48th International Conference on Environmental Systems, vol. 81 (2018)

68. Josan, A.S., Green, A.M., Malik, K.A.: Generalized Constraints on the Curvature Perturbation from Primordial Black Holes. Phys. Rev. D 79, 103520 (2009)

69. Karwal, T., Kamionkowski, M.: Dark Energy at Early Times, the Hubble Parameter, and the String Axiverse. Phys. Rev. D 94, 103523 (2016)

70. Kazantzidis, S., Kravtsov, A.V., Zentner, A.R., et al.: The Effect of Gas Cooling on the Shapes of Dark Matter Halos. ApJ 611, L73 (2004)

71. Kervella, P., Arenou, F., Schneider, J.: Orbital Inclination and Mass of the Exoplanet Candidate Proxima c. A\&A 635, L14 (2020)

72. Kiefer, F.: Determining the Mass of the Planetary Candidate HD 114762 b using Gaia. A\&A 632, L9 (2019)

73. King, A., Nixon, C.: Black Holes in Stellar-Mass Binary Systems: Expiating Original Spin? MNRAS 462, 464 (2016)

74. Koposov, S.E., Boubert, D., Li, T.S., et al.: Discovery of a nearby $1700 \mathrm{~km} \mathrm{~s}^{-1}$ Star Ejected from the Milky Way by Sgr A*. MNRAS 491, 2465 (2020)

75. Kral, Q., Schneider, J., Kennedy, G., Souami, D.: Effects of Disc Asymmetries on Astrometric Measurements. Can they Mimic Planets? A\&A 592, A39 (2016)

76. Lagrange, A.-M., Meunier, N., Desort, M., Malbet, F.: Using the Sun to Estimate Earth-like Planets Detection Capabilities . III. Impact of Spots and Plages on Astrometric Detection. A\&A 528, L9 (2011)

77. Laskar, J., Robutel, P.: The Chaotic Obliquity of the Planets. Nature 361, 608 (1993)

78. Lattimer, J.M., Prakash, M.: Ultimate Energy Density of Observable Cold Baryonic Matter. Phys. Rev. Lett. 94, 111101 (2005)

79. Lattimer, J.M., Prakash, M.: The Equation of State of Hot, Dense Matter and Neutron Stars. Phys. Rep. 621, 127 (2016)

80. Leger, A.M.: Respective capabilities of Affordable Coronagraphs and Interferometers Searching for Biosignatures. In: Pathways Towards Habitable Planets, 99 (2015)

81. Li, F., Erickcek, A.L., Law, N.M.: A New Probe of the Small-Scale Primordial Power Spectrum: Astrometric Microlensing by Ultracompact Minihalos. Phys. Rev. D 86, 043519 (2012)

82. Lovis, C., Dumusque, X., Santos, N.C., et al.: arXiv:1107.5325 (2011)

83. LSST Science Collaboration, Abell, P.A., Allison, J., et al.: arXiv:0912.0201 (2009)

84. Maccarone, T.J.: On the Misalignment of Jets in Microquasars. MNRAS 336, 1371 (2002)

85. Madhusudhan, N., Burrows, A.: Analytic Models for Albedos, Phase Curves, and Polarization of Reflected Light from Exoplanets. ApJ 747, 25 (2012)

86. Malbet, F., Léger, A., Anglada Escudé, G., et al.: Microarcsecond Astrometric Observatory Theia: from Dark Matter to Compact Objects and Nearby Earths. In: Proc. SPIE, vol. 9904, p. 99042F (2016)

87. Malbet, F. et al.: High Precision Astrometry Mission for the Detection and Characterization of Nearby Habitable Planetary Systems with the Nearby Earth Astrometric Telescope (NEAT). Experimental Astronomy 34, 385 (2012)

88. Malhan, K., Ibata, R.A., Carlberg, R.G., Valluri, M., Freese, K.: Butterfly in a Cocoon, Understanding the Origin and Morphology of Globular Cluster Streams: the Case of GD-1. ApJ 881, 106 (2019)

89. Mayor, M., Marmier, M., Lovis, C., et al.: arXiv:1109.2497 (2011)

90. Mayor, M., Queloz, D.: A Jupiter-Mass Companion to a Solar-Type Star. Nature 378, 355 (1995)

91. Merritt, D., Poon, M.Y.: Chaotic Loss Cones and Black Hole Fueling. ApJ 606, 788 (2004)

92. Meru, F., Galvagni, M., Olczak, C.: Growth of Grains in Brown Dwarf Disks. ApJ 774, L4 (2013)

93. Miller, L., Kitching, T.D., Heymans, C., Heavens, A.F., van Waerbeke, L.: Bayesian Galaxy Shape Measurement for Weak Lensing Surveys - I. Methodology and a Fast-Fitting Algorithm. MNRAS 382, 315 (2007)

94. Miller, M.C., Lamb, F.K.: Observational Constraints on Neutron Star Masses and Radii. European Phys. J. A 52, 63 (2016)

95. Mirabel, I.F., Dhawan, V., Mignani, R.P., Rodrigues, I., Guglielmetti, F.: A High-Velocity Black Hole on a Galactic-Halo Orbit in the Solar Neighbourhood. Nature 413, 139 (2001)

96. Necib, L., Lisanti, M., Belokurov, V.: Inferred Evidence for Dark Matter Kinematic Substructure with SDSS-Gaia. ApJ 874, 3 (2019) 
97. Oñorbe, J., Boylan-Kolchin, M., Bullock, J.S., et al.: Forged in FIRE: Cusps, Cores and Baryons in Low-Mass Dwarf Galaxies. MNRAS 454, 2092 (2015)

98. Orosz, J.A., McClintock, J.E., Aufdenberg, J.P., et al.: The Mass of the Black Hole in Cygnus X-1. ApJ 742, 84 (2011)

99. Özel, F., Freire, P.: Masses, Radii, and the Equation of State of Neutron Stars. ARA\&A 54, 401 (2016)

100. Özel, F., Psaltis, D.: Reconstructing the Neutron-Star Equation of State from Astrophysical Measurements. Phys. Rev. D 80, 103003 (2009)

101. Paczyński, B.: Gravitational Microlensing by the Galactic Halo. ApJ 304, 1 (1986)

102. Payne, M.J., Lodato, G.: The Potential for Earth-Mass Planet Formation around Brown Dwarfs. MNRAS 381, 1597 (2007)

103. Perryman, M.A.C., Lindegren, L., Kovalevsky, J., et al.: The HIPPARCOS Catalogue. A\&A 323, L49 (1997)

104. Planck Collaboration, Ade, P.A.R., Aghanim, N., et al.: Planck Early Results. I. The Planck Mission. A\&A 536, A1 (2011)

105. Price-Whelan, A.M., Bonaca, A.: Off the Beaten Path: Gaia Reveals GD-1 Stars Outside of the Main Stream. ApJ 863, L20 (2018)

106. Ranalli, P., Hobbs, D., Lindegren, L.: Astrometry and Exoplanets in the Gaia Era: a Bayesian Approach to Detection and Parameter Recovery. A\&A 614, A30 (2018)

107. Rauer, H., Heras, A.M.: Space Missions for Exoplanet Science: PLATO. In: Deeg, H.J., Belmonte, J.A. (eds.) Handbook of Exoplanets, pp. 1309-1330. Springer International Publishing, Cham (2018)

108. Read, J.I., Agertz, O., Collins, M.L.M.: Dark Matter Cores All the Way Down. MNRAS 459, 2573 (2016)

109. Refsdal, S.: On the Possibility of Determining Hubble's Parameter and the Masses of Galaxies from the Gravitational Lens Effect. MNRAS 128, 307 (1964)

110. Remillard, R.A., McClintock, J.E.: X-Ray Properties of Black-Hole Binaries. ARA\&A 44, 49 (2006)

111. Ricci, L., Testi, L., Natta, A., Scholz, A., de Gregorio-Monsalvo, I.: ALMA Observations of $\rho$-Oph 102: Grain Growth and Molecular Gas in the Disk around a Young Brown Dwarf. ApJ 761, L20 (2012)

112. Ricci, L., Testi, L., Natta, A., et al.: Brown Dwarf Disks with ALMA. ApJ 791, 20 (2014)

113. Ricker, G.R., Winn, J.N., Vanderspek, R., et al.: Transiting Exoplanet Survey Satellite (TESS). J. Astron. Telesc. Instrum. Syst 1, 014003 (2015)

114. Ricotti, M., Gould, A.: A New Probe of Dark Matter and High-Energy Universe Using Microlensing. ApJ 707, 979 (2009)

115. Riess, A.G., Macri, L.M., Hoffmann, S.L., et al.: A $2.4 \%$ Determination of the Local Value of the Hubble Constant. ApJ 826, 56 (2016)

116. Rusu, C.E., Fassnacht, C.D., Sluse, D., et al.: H0LiCOW - III. Quantifying the Effect of Mass along the Line of Sight to the Gravitational Lens HE 0435-1223 through Weighted Galaxy Counts. MNRAS 467, 4220 (2017)

117. Satyal, S., Musielak, Z.E.: Stability of a Planet in the HD 41004 Binary System. Astronomische Nachrichten 337, 300 (2016)

118. Schaeffer, R., Silk, J.: Massive Neutrinos and the Pancake Theory of Galaxy Formation. Astrophys. J. 281, L13 (1984)

119. Scheidel, D.: 2011 journées de Telemetrie Laser OCA (2011)

120. Scholz, A., Jayawardhana, R., Wood, K., et al.: IRAS 04325+2402C: A Very Low Mass Object with an Edge-On Disk. ApJ 681, L29 (2008)

121. Schwieterman, E.W., Meadows, V.S., Domagal-Goldman, S.D., et al.: Identifying Planetary Biosignature Impostors: Spectral Features of $\mathrm{CO}$ and $\mathrm{O}_{4}$ Resulting from Abiotic $\mathrm{O}_{2} / \mathrm{O}_{3}$ Production. ApJ 819, L13 (2016)

122. Shabram, M.I., Batalha, N., Thompson, S.E., et al.: Sensitivity Analyses of Exoplanet Occurrence Rates from Kepler and Gaia. AJ 160, 16 (2020)

123. Shen, K.J., Boubert, D., Gänsicke, B.T., et al.: Three Hypervelocity White Dwarfs in Gaia DR2: Evidence for Dynamically Driven Double-degenerate Double-detonation Type Ia Supernovae. ApJ $\mathbf{8 6 5}, 15$ (2018)

124. Simms, L.: Hybrid CMOS SI PI Detectors as Astronomical Imagers. PhD Dissertation, Stanford University (2009) 
125. Sluse, D., Sonnenfeld, A., Rumbaugh, N., et al.: H0LiCOW - II. Spectroscopic Survey and GalaxyGroup Identification of the Strong Gravitational Lens System HE 0435-1223. MNRAS 470, 4838 (2017)

126. Snellen, I., de Kok, R., Birkby, J.L., et al.: Combining High-Dispersion Spectroscopy with high Contrast Imaging: Probing Rocky Planets around our Nearest Neighbors. A\&A 576, A59 (2015)

127. Sozzetti, A.: Gaia, Non-Single Stars, Brown Dwarfs, and Exoplanets. Mem Soc. Astron. Italiana 85, 643 (2014)

128. Sozzetti, A., de Bruijne, J.: Handbook of Exoplanets. In: Deeg, H.J., Belmonte, J.A. (eds.), pp. 12051228. Springer International Publishing, Cham (2018)

129. Spergel, D.N., Steinhardt, P.J.: Observational Evidence for Self-Interacting Cold Dark Matter. Phys. Rev. Lett. 84, 3760 (2000)

130. Suyu, S.H., Auger, M.W., Hilbert, S., et al.: Two Accurate Time-delay Distances from Strong Lensing: Implications for Cosmology. ApJ 766, 70 (2013)

131. Suyu, S.H., Bonvin, V., Courbin, F., et al.: HOLiCOW - I. $\mathrm{H}_{0}$ Lenses in COSMOGRAIL's Wellspring: Program Overview. MNRAS 468, 2590 (2017)

132. Suyu, S.H., Treu, T., Hilbert, S., et al.: Cosmology from Gravitational Lens Time Delays and Planck Data. ApJL 788, L35 (2014)

133. Thebault, P.: Against All Odds? Forming the Planet of the HD 196885 Binary. Celest. Mech. Dyn. Astron. 111, 29 (2011)

134. Thebault, P., Haghighipour, N.: Planet formation in binaries. Springer geophysics, pp. 309-340 (2015)

135. Tomsick, J.A., Muterspaugh, M.W.: Masses of Neutron Stars in High-mass X-ray Binaries with Optical Astrometry. ApJ 719, 958 (2010)

136. Tomsick, J.A., Nowak, M.A., Parker, M., et al.: The Reflection Component from Cygnus X-1 in the Soft State Measured by NuSTAR and Suzaku. ApJ 780, 78 (2014)

137. Udalski, A., Jung, Y.K., Han, C., et al.: A Venus-mass Planet Orbiting a Brown Dwarf: A Missing Link between Planets and Moons. ApJ 812, 47 (2015)

138. Valluri, M., Debattista, V.P., Quinn, T., Moore, B.: The Orbital Evolution Induced by Baryonic Condensation in Triaxial Haloes. MNRAS (V10) 403, 525 (2010)

139. Valluri, M., Debattista, V.P., Quinn, T.R., Roškar, R., Wadsley, J.: Probing the Shape and History of the Milky Way Halo with Orbital Spectral Analysis. MNRAS 419, 1951 (2012)

140. Valluri, M., Debattista, V.P., Stinson, G.S., et al.: Halo Orbits in Cosmological Disk Galaxies: Tracers of Formation History. ApJ 767, 93 (2013)

141. Valluri, M., Debattista, V.P., Stinson, G.S., et al.: Halo Orbits in Cosmological Disk Galaxies: Tracers of Formation History. ApJ 767, 93 (2013)

142. Walker, M.G., Mateo, M., Olszewski, E.W., et al.: A Universal Mass Profile for Dwarf Spheroidal Galaxies? ApJ 704, 1274 (2009)

143. Walker, M.G., Peñarrubia, J.: A Method for Measuring (Slopes of) the Mass Profiles of Dwarf Spheroidal Galaxies. ApJ 742, 20 (2011)

144. Walton, D.J., Tomsick, J.A., Madsen, K.K., et al.: The Soft State of Cygnus X-1 Observed with NuSTAR: a Variable Corona and a Stable Inner Disk. ApJ 826, 87 (2016)

145. Watkins, L.L., van de Ven, G., den Brok, M., van den Bosch, R.C.E.: Discrete Dynamical Models of $\omega$ Centauri. MNRAS 436, 2598 (2013)

146. Weber, F.: Strangeness in Neutron Stars. J. Phys. G Nuclear Particle Phys. 27, 465 (2001)

147. Widrow, L.M., Gardner, S., Yanny, B., Dodelson, S., Chen, H.-Y.: Galactoseismology: Discovery of Vertical Waves in the Galactic Disk. ApJ 750, L41 (2012)

148. Winn, J.N., Fabrycky, D.C.: The Occurrence and Architecture of Exoplanetary Systems. ARA\&A 53, 409 (2015)

149. Wolf, J., Martinez, G.D., Bullock, J.S., et al.: Accurate Masses for Dispersion-Supported Galaxies. MNRAS 406, 1220 (2010)

150. Wong, K.C., Suyu, S.H., Auger, M.W., et al.: H0LiCOW - IV. Lens Mass Model of HE 0435-1223 and Blind Measurement of its Time-Delay Distance for Cosmology. MNRAS 465, 4895 (2017)

151. Wong, K.C., Suyu, S.H., Chen, G.C.F., et al.: H0LiCOW - XIII. A 2.4\% Measurement of $\mathrm{H}_{0}$ from Lensed Quasars: 5.3 $\sigma$ Tension Between Early- and Late-Universe p. MNRAS 498, 1420 (2020)

152. Xuan, J.W., Wyatt, M.C.: Evidence for a High Mutual Inclination Between the Cold Jupiter and Transiting Super Earth Orbiting $\pi$ Men. MNRAS 497, 2096 (2020) 
153. Yu, Q., Madau, P.: Kinematics of Hypervelocity Stars in the Triaxial Halo of the Milky Way. MNRAS 379, 1293 (2007)

154. Yu, Q., Tremaine, S.: Ejection of Hypervelocity Stars by the (Binary) Black Hole in the Galactic Center. ApJ 599, 1129 (2003)

155. Zarka, P., Lazio, J., Hallinan, G.: Magnetospheric Radio Emissions from Exoplanets with the SKA. In: Advancing Astrophysics with the Square Kilometre Array (AASKA14), 120 (2015)

156. Zemp, M., Gnedin, O.Y., Gnedin, N.Y., Kravtsov, A.V.: The Impact of Baryon Physics on the Structure of High-Redshift Galaxies. ApJ 748, 54 (2012)

Publisher's note Springer Nature remains neutral with regard to jurisdictional claims in published maps and institutional affiliations. 


\section{Affiliations}

Fabien Malbet ${ }^{1}$ (D) . Céline Boehm ${ }^{2}$. Alberto Krone-Martins ${ }^{3}$. Antonio Amorim ${ }^{4}$. Guillem Anglada-Escudé ${ }^{5}$. Alexis Brandeker ${ }^{6}$. Frédéric Courbin ${ }^{7} \cdot$ Torsten Enßlin $^{8} \cdot$ Antonio Falcão $^{9} \cdot$ Katherine Freese $^{6,10}$. Berry Holl ${ }^{11}$. Lucas Labadie $^{12}$. Alain Léger ${ }^{13}$. Gary A. Mamon ${ }^{14}$.

Barbara McArthur ${ }^{10}$. Alcione Mora ${ }^{15}$. Mike Shao ${ }^{16}$. Alessandro Sozzetti ${ }^{17}$. Douglas Spolyar ${ }^{6}$. Eva Villaver ${ }^{18}$. Ummi Abbas ${ }^{17}$. Conrado Albertus ${ }^{19}$. João Alves ${ }^{20}$. Rory Barnes ${ }^{21}$. Aldo Stefano Bonomo ${ }^{17}$. Hervé Bouy ${ }^{22}$. Warren R. Brown ${ }^{23}$. Vitor Cardoso ${ }^{24} \cdot$ Marco Castellani $^{25}$. Laurent Chemin ${ }^{26}$. Hamish Clark ${ }^{27}$. Alexandre C. M. Correia ${ }^{28}$. Mariateresa Crosta ${ }^{17}$.

Antoine Crouzier ${ }^{29}$. Mario Damasso ${ }^{17}$. Jeremy Darling ${ }^{30}$. Melvyn B. Davies ${ }^{31}$. Antonaldo Diaferio ${ }^{32,33} \cdot$ Morgane Fortin $^{34}$. Malcolm Fridlund ${ }^{35,36}$. Mario Gai ${ }^{17}$. Paulo Garcia ${ }^{37}$. Oleg Gnedin ${ }^{38}$. Ariel Goobar ${ }^{6}$. Paulo Gordo ${ }^{39}$. Renaud Goullioud ${ }^{16}$. David Hall ${ }^{40}$. Nigel Hambly ${ }^{41}$. Diana Harrison ${ }^{42}$.

David Hobbs $^{43}$. Andrew Holland ${ }^{40}$. Erik Høg ${ }^{44}$. Carme Jordi ${ }^{45}$.

Sergei Klioner ${ }^{46}$. Ariane Lançon $^{47}$. Jacques Laskar ${ }^{48}$. Mario Lattanzi ${ }^{17}$. Christophe Le Poncin-Lafitte ${ }^{49}$. Xavier Luri ${ }^{45}$. Daniel Michalik ${ }^{50}$.

André Moitinho de Almeida ${ }^{51}$. Ana Mourão ${ }^{24}$. Leonidas Moustakas ${ }^{16}$.

Neil J. Murray ${ }^{40}$. Matthew Muterspaugh ${ }^{52}$. Micaela Oertel ${ }^{53}$.

Luisa Ostorero ${ }^{32,33}$. Jordi Portell ${ }^{45}$. Jean-Pierre Prost ${ }^{54}$.

Andreas Quirrenbach ${ }^{55}$. Jean Schneider ${ }^{53}$. Pat Scott ${ }^{56,57}$. Arnaud Siebert ${ }^{47}$. Antonio da Silva ${ }^{58} \cdot$ Manuel Silva ${ }^{59}$. Philippe Thébault ${ }^{29} \cdot$ John Tomsick $^{60}$. Wesley Traub ${ }^{16}$. Miguel de Val-Borro ${ }^{61}$. Monica Valluri ${ }^{38}$.

Nicholas A. Walton ${ }^{62}$. Laura L. Watkins ${ }^{63}$. Glenn White ${ }^{64,65}$.

Lukasz Wyrzykowski $^{66} \cdot$ Rosemary Wyse $^{67} \cdot$ Yoshiyuki Yamada $^{68}$

Univ. Grenoble Alpes, CNRS, IPAG, Grenoble, France

2 The University of Sydney, School of Physics, Camperdown, Australia

3 CENTRA/University of Lisbon, Lisbon, Portugal

4 FCUL, CENTRA/SIM, Lisbon, Portugal

5 Queen Mary University of London, London, UK

6 Stockholm University, Stockholm, Sweden

7 EPFL, Lausanne, Switzerland

8 Max-Planck Institute for Astrophysics, Garching, Germany

9 Uninova, Caparica, Portugal

10 University of Texas, Austin, TX, USA

11 Department of Astronomy, University of Geneva, Geneva, Switzerland

12 University of Cologne, Cologne, Germany

13 Univ. Paris-Saclay, CNRS, Institut d'astrophysique spatiale, Orsay, France

14 Institut d'Astrophysique de Paris (UMR 7095: CNRS, Sorbonne Université), Paris, France

15 Aurora Technology BV, Madrid, Spain 
Jet Propulsion Laboratory, California Institute of Technology, Pasadena, CA, USA

Obs. Torino/INAF, Pino Torinese, Italy

Centro de Astrobiología (CAB, CSIC-INTA), ESAC Campus, s/n/, 28692 Villanueva de la Cañada, Madrid, Spain

Universidad de Granada, Granada, Spain

University of Vienna, Vienna, Austria

Astronomy Department, University of Washington, Seattle, WA, USA

Laboratoire d'Astrophysique de Bordeaux, University of Bordeaux, CNRS, Pessac, France

CfA - Harvard, Smithsonian, Cambridge, MA, USA

CENTRA, IST, Universidade de Lisboa, Lisbon, Portugal

INAF - Rome Astronomical Obs., Roma, Italy

University of Antofogasta, Antofogasta, Chile

University of Sydney, Sydney, Australia

CFisUC, Department of Physics, University of Coimbra, Coimbra, Portugal

LESIA, Observatoire de Paris, Université PSL, Sorbonne Université, Université de Paris, CNRS, Meudon, France

University of Colorado, Boulder, CO, USA

Lund University, Lund, Sweden

Dipartimento di Fisica, Università di Torino, Torino, Italy

Istituto Nazionale di Fisica Nucleare (INFN), Sezione di Torino, Torino, Italy

Copernicus Astronomical Center, Warsaw, Poland

Leiden Obs., Leiden, The Netherlands

Chalmers University, Gothenburg, Sweden

CENTRA, FEUP, University of Porto, Porto, Portugal

University of Michigan, Ann Arbor, MI, USA

University of Lisbon - CENTRA/SIM, Lisbon, Portugal

The Open University, Milton Keynes, UK

University of Edinburgh, Edinburgh, UK

IoA, Cambridge, UK

Lund Observatory, Department of Astronomy and Theoretical Physics, Lund University, Lund, Sweden

Niels Bohr Institute, Copenhague, Denmark

Dept. FQA, Institut de Ciències del Cosmos (ICCUB), Universitat de Barcelona (IEEC-UB), Martí Franquès 1, E08028 Barcelona, Spain

Lohrmann Obs., Technische Universität Dresden, Dresden, Germany

University of Strasbourg, CNRS, Obs. Astron. Strasbourg, Strasbourg, France

IMCCE, Observatoire de Paris, Université PSL, Sorbonne Université, CNRS, Paris, France

SYRTE, Observatoire de Paris, Université PSL, CNRS, Sorbonne Université, LNE, 61 avenue de l'Observatoire 75014 Paris, France

ESA/ESTEC, Noordwijk, The Netherlands 
51 CENTRA, Faculdade de Ciências, Universidade de Lisboa, Campo Grande, 1749-016 Lisbon, Portugal

52 Dean of Science, Technology, and Mathematics, Columbia State Community College, Columbia, TN, USA

53 LUTH, Observatoire de Paris, Université PSL, CNRS, Université de Paris, Meudon, France

54 Thales Alenia Space, Cannes, France

55 University of Heidelberg, Heidelberg, Germany

56 University of Queensland, Brisbane, Australia

57 Imperial College London, London, UK

58 Instítuto de Astrofísica e Ciências do Espaço, Faculdade de Ciências, Universidade de Lisboa, Lisboa, Portugal

59 CENTRA/SIM-FEUP, University of Porto, Porto, Portugal

60 SSL Berkeley, Berkeley, CA, USA

61 Planetary Science Institute, Tucson, AZ, USA

62 Institute of Astronomy, University of Cambridge, Cambridge, UK

63 AURA for the European Space Agency, ESA Office, Space Telescope Science Institute, 3700 San Martin Drive, 21218 Baltimore, MD, USA

64 RAL Space, STFC Rutherford Appleton Laboratory, Chilton, Didcot, Oxfordshire, OX11 0QX, UK

65 Department of Physics and Astronomy, The Open University, Walton Hall, Milton Keynes, MK7 6AA, UK

66 Astronomical Observatory, University of Warsaw, Warsaw, Poland

67 Johns Hopkins University, Baltimore, MD, USA

68 Department of Physics, Kyoto University, Kyoto, Japan 